\title{
Local tube realizations of CR-manifolds and maximal Abelian subalgebras
}

\author{
GREGOR FELS AND WiLHELM KAUP
}

\begin{abstract}
For every real-analytic CR-manifold $M$ we give necessary and sufficient conditions that $M$ can be realized in a suitable neighbourhood of a given point $a \in M$ as a tube submanifold of some $\mathbb{C}^{r}$. We clarify the question of the 'right' equivalence between two local tube realizations of the CR-manifold germ $(M, a)$ by introducing two different notions of affine equivalence. One of our key results is a procedure that reduces the classification of equivalence classes to a purely algebraic manipulation in terms of Lie theory.
\end{abstract}

Mathematics Subject Classification (2010): 32V05 (primary); 32V40, 32M25, 17B66 (secondary).

\section{Introduction}

Among all CR-submanifolds of $\mathbb{C}^{r}$ a special class is formed by the tube submanifolds, that is, by real submanifolds of the form

$$
T_{F}=\mathbb{R}^{r}+i F
$$

with $F$ an arbitrary submanifold of $\mathbb{R}^{r}$, called the base of $T_{F}$. CR-manifolds of this type play a fundamental role in CR-geometry as they often serve as test objects. In addition, the interplay between real geometric properties of the base $F$ and CRproperties of the associated tube $T_{F}$ are quite fruitful. An early example of this interplay is well known in the case of open tube submanifolds: The tube domain $T_{F} \subset \mathbb{C}^{r}$ is holomorphically convex if and only if the (open) base $F \subset \mathbb{R}^{r}$ is convex in the elementary sense. Clearly, in the context of CR-geometry, domains in $\mathbb{C}^{r}$ are not of interest. In fact, we will mainly consider CR-manifolds $M=$ $(M, H M, J)$ which are holomorphically nondegenerate, i.e., $\xi=0$ is the only local holomorphic vector field on $M$, which is a section in the subbundle $H M$. We note in passing that in the tube situation the general case can be reduced to the nondegenerate one as every such CR-manifold is locally a direct product of some $\mathbb{C}^{k}$ and a holomorphically nondegenerate CR-manifold.

Received September 18, 2009; accepted January 14, 2010. 
For instance, interesting examples of holomorphically nondegenerate tube submanifolds are obtained as follows: Let $\Omega \subset \mathbb{R}^{r}$ be an open convex cone such that the corresponding tube domain $T_{\Omega} \subset \mathbb{C}^{r}$ is biholomorphically equivalent to an irreducible bounded symmetric domain. Then the group $G=\mathrm{GL}(\Omega):=\{\mathrm{g} \in$ $\mathrm{GL}(\mathrm{r}, \mathbb{R}): \mathrm{g}(\Omega)=\Omega\}$ acts transitively on $\Omega$ and for every non-open $G$-orbit $F \subset \mathbb{R}^{r}$ with $F \neq\{0\}$ the corresponding tube $T_{F}$ is Levi degenerate but still is holomorphically nondegenerate [18]. The example of lowest possible dimension occurs with the future cone $\Omega=\left\{x \in \mathbb{R}^{3}: x_{3}>\sqrt{x_{1}^{2}+x_{2}^{2}}\right\}$ in 3-dimensional spacetime and $F=\left\{x \in \mathbb{R}^{3}: x_{3}=\sqrt{x_{1}^{2}+x_{2}^{2}}>0\right\}$ the future light cone. The future light cone tube $T_{F}$ has been studied by many authors and has remarkable properties, $c f$. [9] and the references therein. Until recently, this tube manifold $T_{F}$ was, up to local CR-isomorphy, the only known example of a 5-dimensional Levi degenerate, holomorphically nondegenerate and locally homogeneous CR-manifold. A full classification of CR-manifolds of this type could be obtained in [10] - surprisingly all possible examples turned out to be locally representable as tube manifolds.

Since tube manifolds are quite easy to deal with it is of interest to decide whether a given CR-manifold $M$ is CR-isomorphic, at least locally around a given point $a \in M$, to a tube submanifold of some $\mathbb{C}^{r}$. Another question is how many 'different' tube realizations a given CR-manifold germ does admit. In the particular case of spherical hypersurfaces the following result has been obtained in [7] by solving a certain partial differential equation coming from the Chern-Moser theory [6]: For every $r \geq 2$ there exist, up to affine equivalence, precisely $r+2$ closed smooth tube submanifolds of $\mathbb{C}^{r}$ that are locally CR-isomorphic to the Euclidean sphere $S^{2 r-1} \subset \mathbb{C}^{r}$. In $[12,13]$ the same method has been used for a certain more general class of CR-flat manifolds. All the above results rely on Chern-Moser theory and therefore only apply to CR-manifolds that are Levi nondegenerate and of hypersurface type.

In this note we use a different method that applies to all CR-manifolds (for simplicity we work in the category of real-analytic CR-manifolds). This method is more algebraic in nature and starts from the following simple observation: A real submanifold $M \subset \mathbb{C}^{r}$ is tube (1.1) if and only if $M$ is invariant under all real translations $z \mapsto z+v$ with $v \in \mathbb{R}^{r}$. In particular, $\mathfrak{g}:=\mathfrak{h o l}(M, a)$, the Lie algebra of all (germs of real-analytic) infinitesimal CR-transformations at $a$, contains the Abelian Lie subalgebra induced by the above translations. Therefore it is not unexpected that every tube realization of an arbitrarily given CR-manifold germ $(M, a)$ is strongly related to a certain Abelian Lie subalgebra $\mathfrak{v}$ of $\mathfrak{h o l}(M, a)$, see Proposition 4.1 and Proposition 4.3 for precise statements.

In a slightly different form the Lie algebra $\mathfrak{v}$ has already been used in [1] for the characterization of tube manifolds (in fact more generally in the context of abstract smooth CR-manifolds and the solution of the local integrability problem for rigid CR-manifolds; on the other hand we do not need to assume that the evaluation map $\varepsilon_{a}: \mathfrak{v} \rightarrow T_{a} M$ is injective). But, in contrast to [1] our intentions are completely different -we mainly focus on the question of how many 'essentially' different tube 
realizations of a given CR-manifold germ $(M, a)$ do exist. This question of equivalence for different local tube realizations of a given CR-manifold is a bit more subtle than it might appear at the first glance. We introduce two different notions of equivalence to which we refer accordingly as to the 'strict' and the 'coarse' affine equivalence. Our impression is that the latter one is more appropriate in the context of local tube realizations.

In Section 4 we give necessary and sufficient conditions for an Abelian subalgebra $\mathfrak{v} \subset \mathfrak{g}$ to give a local tube realization of $(M, a)$. This characterization also includes for every $\mathfrak{v}$ an easy to compute canonical form of a local CR-isomorphism to the corresponding tube realization of $(M, a)$. It is also shown that any two local tube realizations of the germ $(M, a)$ are affinely equivalent (in the strict sense) if and only if the corresponding Abelian subalgebras $\mathfrak{v}, \mathfrak{v}^{\prime} \subset \mathfrak{g}$ are conjugate with respect to the stability group Aut $(M, a)$.

The 'coarse' equivalence relation for tube realizations of the germ $(M, a)$ is, roughly speaking, defined as follows: Two tube realizations $(T, c),\left(T^{\prime}, c^{\prime}\right)$ of $(M, a)$ in $\mathbb{C}^{r}$ are considered to be equivalent in this broader sense if the representing tube submanifolds $T, T^{\prime} \subset \mathbb{C}^{r}$ can be chosen in such a way that $T^{\prime}=g(T)$ for some affine isomorphism $g$ on $\mathbb{C}^{r}$ (that is, without requiring $c^{\prime}=g(c)$ in addition).

While it is not surprising that the existence of a tube realization for $(M, a)$ is closely related to the existence of a certain 'big' Abelian Lie subalgebra of $\mathfrak{g}=\mathfrak{h o l}(M, a)$, it is not at all clear what the relation between various tube realizations and the corresponding Abelian subalgebras in $\mathfrak{h o l}(M, a)$ should be. One of our main results is then obtained in Section 7, where we introduce the subgroup Glob $(M, a) \subset$ Aut $(\mathfrak{g})$ and show for a large class of CR-manifolds $M$ that the local tube realizations of $(M, a)$ are equivalent in the coarser sense if and only if the corresponding Abelian subalgebras $\mathfrak{v}, \mathfrak{v}^{\prime}$ are conjugate with respect to the group $\operatorname{Glob}(M, a)$.

In Sections 8 and 9 we apply our general theory to some concrete cases. For instance, we relate the results from [7] with our algebraic point of view, and identify the various Abelian subalgebras of $\mathfrak{h o l}\left(S^{2 r-1}, a\right), S^{2 r-1} \subset \mathbb{C}^{r}$ the standard sphere, which correspond to various defining equations in [7].

In the last two sections we generalize the notion of a tube submanifold to the notion of a Siegel submanifold. This is motivated by the well known fact that every bounded homogeneous domain can be realized as a Siegel domain, thus giving a lot of additional insight to the structure of those domains. In the forthcoming paper [11] our method will be applied to the class of all Levi non-degenerate real hyperquadrics in $\mathbb{C}^{r}$ in order to obtain a full algebraic characterization of local tube realizations in such cases.

\section{Preliminaries and notation}

Abstract CR-manifolds. A triple $(M, H M, J)$ is called an (abstract) CR-manifold (CR stands for Cauchy-Riemann) if $M$ is a (connected if not stated otherwise explicitly) smooth manifold, $H M$ is a smooth subbundle of its tangent bundle $T M$ 
and $J$ is a smooth bundle endomorphism of $H M$ with $J^{2}=-\mathrm{id}$. For simplicity we often write just $M$ instead of $(M, H M, J)$. For every $a \in M$ the restriction of $J$ to the linear subspace $H_{a} M \subset T_{a} M$ makes $H_{a} M$ to a complex vector space, we call it the holomorphic tangent space to $M$ at a (in the literature $H_{a} M$ is also called the complex tangent space and denoted by $\left.T_{a}^{c} M\right)$. Its complex dimension is called the $C R$-dimension and the real dimension of $T_{a} M / H_{a} M$ is called the $C R$-codimension of $M$. With $M=(M, H M, J)$ also $M^{\text {conj }}:=(M, H M,-J)$ is a CR-manifold; we call it the conjugate of $M$.

A smooth map $g: M \rightarrow M^{\prime}$ between two CR-manifolds is called CR if for every $a \in M$ and $a^{\prime}:=g(a)$ the differential $d g_{a}: T_{a} M \rightarrow T_{a^{\prime}} M^{\prime}$ maps the corresponding holomorphic subspaces in a complex linear way to each other. Also, $g$ is called anti-CR if $g$ is CR as a map $M^{\text {conj }} \rightarrow M^{\prime}$.

For every smooth vector field $\xi$ on $M$ and every $a \in M$ we denote by $\xi_{a} \in$ $T_{a} M$ the corresponding tangent vector at $a$. Furthermore, $\xi$ is called an infinitesimal $C R$-transformation of $M$ if the corresponding local flow on $M$ consists of CR-transformations. With $\xi, \eta$ also the usual bracket $[\xi, \eta]$ is an infinitesimal CRtransformation.

It is obvious that every smooth manifold $M$ can be considered as a CR-manifold with CR-dimension 0 (these are called the totally real CR-manifolds). The other extreme is formed by the CR-manifolds with CR-codimension 0, these are precisely the almost complex manifolds. Among the latter the integrable ones play a special role, the complex manifolds. CR-mappings between complex manifolds are precisely the holomorphic mappings.

CR-manifolds in this paper are understood to be those $M=(M, H M, J)$ that are real-analytic and integrable in the following sense: $M$ is a real-analytic manifold and there is a complex manifold $Z$ such that $M$ can be realized as a real-analytic submanifold $M \subset Z$ with $H_{a} M=T_{a} M \cap i T_{a} M$ and $J(\xi)=i \xi$ for every $a \in M$, $\xi \in H_{a} M$, where $T_{a} M$ is considered in the canonical way as an $\mathbb{R}$-linear subspace of the complex vector space $T_{a} Z$. This notion of integrability is equivalent to the vanishing of the restricted Nijenhuis tensor. We refer to [5] or [3] for further details. The embedding $M \subset Z$ above can always be chosen to be generic, that is, $T_{a} Z=T_{a} M+i T_{a} M$ for all $a \in M$. In that case the (connected) complex manifold $Z$ has complex dimension (CR-dim $M+C R$-codim $M$ ).

CR-isomorphisms between CR-manifolds are always understood to be analytic in both directions. In particular, Aut $(M)$ is the group of all (bianalytic) CRautomorphisms of $M$ and $\operatorname{Aut}_{a}(M):=\{g \in \operatorname{Aut}(M): g(a)=a\}$ is the isotropy subgroup at the point $a \in M$. With $\operatorname{Aut}(M, a)$, also called the stability group at $a$, we denote the group of all CR-automorphisms of the manifold germ $(M, a)$. Then Aut $a(M)$ can be considered in a canonical way as a subgroup of Aut $(M, a)$.

With $\mathfrak{h o l}(M)$ we denote the space of all real-analytic infinitesimal transformations of the CR-manifold $M$ and with $\mathfrak{h o l}(M, a)$ the space of all germs at $a \in M$ of vector fields $\xi \in \mathfrak{h o l}(N)$ where $N$ runs through all open connected neighbourhoods of $a$ in $M$. Then $\mathfrak{h o l}(M)$ as well as every $\mathfrak{h o l}(M, a)$ together with the bracket $[$, ] is a real Lie algebra (of possibly infinite dimension). The canonical restriction 
mapping $\rho_{a}: \mathfrak{h o l}(M) \rightarrow \mathfrak{h o l}(M, a)$ is an injective homomorphism of Lie algebras. Every isomorphism $g:(M, a) \rightarrow\left(M^{\prime}, a^{\prime}\right)$ of CR-manifold germs induces in a canonical way a Lie algebra homomorphism $g_{*}: \mathfrak{h o l}(M, a) \rightarrow \mathfrak{h o l}\left(M^{\prime}, a^{\prime}\right)$. Its inverse is the pull back $g^{*}$. Clearly, $g \mapsto g_{*}$ defines a group homomorphism Ad : Aut (M, a) $\rightarrow$ Aut $(\mathfrak{h o l}(\mathbf{M}, \mathrm{a}))$.

A vector field $\xi \in \mathfrak{h o l}(M)$ is called complete on $M$ if the corresponding local flow extends to a one-parameter group $\mathbb{R} \rightarrow$ Aut $(M)$. The image of $1 \in \mathbb{R}$ is denoted by $\exp (\xi)$. In this sense we have the exponential map exp : aut $(M) \rightarrow$ Aut $(M)$, where $\mathfrak{a u t}(M)$ is the set of all complete $\xi \in \mathfrak{h o l}(M)$. In general, $\mathfrak{a u t}(M) \subset$ $\mathfrak{h o l}(M)$ is neither a linear subspace nor closed under taking brackets. But, if there exists a Lie subalgebra $\mathfrak{g} \subset \mathfrak{h o l}(M)$ of finite dimension with $\mathfrak{a u t}(M) \subset \mathfrak{g}$, then $\mathfrak{a u t}(M)$ itself is a Lie subalgebra [19] and on Aut $(M)$ there exists a unique Lie group structure (in general not connected) such that exp is a local diffeomorphism in a neighbourhood of $0 \in \mathfrak{a u t}(M)$. Furthermore, the map Aut $(M) \times M \rightarrow M$, $(g, a) \mapsto g(a)$, is real-analytic.

In case $M$ is generically embedded as a real-analytic CR-submanifold of a complex manifold $Z$ then a vector field $\xi$ on $M$ is in $\mathfrak{h o l}(M)$ if and only if $\xi$ has an extension $\widetilde{\xi}$ to a holomorphic vector field on a suitable open neighbourhood $U$ of $M$ in $Z$ (that is, $\widetilde{\xi}$ is a holomorphic section over $U$ in its tangent bundle $T U$ ). The Lie algebras $\mathfrak{h o l}(Z)$ and $\mathfrak{h o l}(Z, a)$ are complex Lie algebras and $\mathfrak{g}:=\mathfrak{h} \mathfrak{o l}(M, a)$ is in a canonical way a real subalgebra of $\mathfrak{h o l}(Z, a)$. The CR-manifold germ $(M, a)$ is called holomorphically nondegenerate if $\mathfrak{g}$ is totally real in $\mathfrak{h o l}(Z, a)$, that is, $\mathfrak{g} \cap i \mathfrak{g}=\{0\}$. In this case there is a unique antilinear Lie algebra automorphism $\sigma$ of $\mathfrak{g}^{\mathbb{C}}:=\mathfrak{g}+i \mathfrak{g} \subset \mathfrak{h o l}(Z, a)$ with $\mathfrak{g}=$ Fix $(\sigma)$. Clearly, real Lie subalgebras of $\mathfrak{g}$ and $\sigma$-invariant complex Lie subalgebras of $\mathfrak{g}^{\mathbb{C}}$ are in a natural 1-1-correspondence.

In general, a vector field $\xi \in \mathfrak{h o l}(M)$ only can be integrated to a local 1parameter group of CR-transformations $g_{t}$ that we also denote by $\exp (t \xi)$. The reason for this notation in the analytic case is the following: To every $a \in M$ and every open neighbourhood $W$ of $a \in Z$ there is a further open neighbourhood $U \subset$ $W$ of $a \in Z$ and an $\varepsilon>0$ such that the $g_{t}$ are defined as holomorphic mappings $U \rightarrow W$ for $|t|<\varepsilon$ and satisfy for every holomorphic mapping $f: W \rightarrow \mathbb{C}^{n}$ the formula

$$
\left.f \circ g_{t}\right|_{U}=\sum_{k=0}^{\infty} \frac{1}{k !}(t \xi)^{k}\left(\left.f\right|_{U}\right) .
$$

In particular, if $f$ gives a local chart for $Z$ around $a$ then the $g_{t}$ on $U$ can be recovered from the right side of this formula.

Lemma 2.1. Let $Z$ be a connected complex manifold of dimension $n$ and $\mathfrak{e} \subset$ $\mathfrak{h o l}(Z, a)$ an Abelian complex Lie subalgebra with $\varepsilon_{a}(\mathfrak{e})=T_{a} Z$, where $\varepsilon_{a}$ is the evaluation map $\xi \mapsto \xi_{a}$. Then $\varepsilon_{a}$ induces a complex linear isomorphism from e onto $T_{a} Z$. In particular, $\mathfrak{e}$ also has dimension $n$ and is maximal Abelian in $\mathfrak{h o l}(Z, a)$.

Proof. Let $\eta \in \mathfrak{e}$ be an arbitrary element with $\eta_{a}=0$. We have to show $\eta=0$. Fix a linear subspace $\mathfrak{a} \subset \mathfrak{e}$ such that $\varepsilon_{a}: \mathfrak{a} \rightarrow T_{a} Z$ is an isomorphism. We may 
assume that $\mathfrak{a} \subset \operatorname{hol}(U)$ for some open neighbourhood $U \subset Z$ of $a$ and also that every $z \in U$ is of the form $z=\exp (\xi)(a)$ for some $\xi \in \mathfrak{a}$. For every such $z$ then $[\eta, \xi]=0$ implies $\exp (t \eta)(z)=\exp (\xi) \exp (t \eta)(a)=\exp (\xi)(a)=z$ for $|t|$ small, that is, $\eta=0$.

For the sake of clarity we mention that in case $n=\operatorname{dim} Z \geq 2$ there exist Abelian subalgebras $\mathfrak{e} \subset \mathfrak{h o l}(Z, a)$ of arbitrary dimension. However, in general these do not span $T_{a} Z$.

The CR-manifold $M$ is called homogeneous if the group Aut $(M)$ acts transitively on $M$. Also, $M$ is called locally homogeneous if for every $a, b \in M$ the manifold germs $(M, a),(M, b)$ are CR-isomorphic. By [21] this is equivalent to $\varepsilon_{a}(\mathfrak{h o l}(M, a))=T_{a} M$ for every $a \in M$. The CR-manifold $M$ is called minimal if every smooth submanifold $N \subset M$ with $H_{a} M \subset T_{a} N$ for all $a \in N$ is already open in $M$.

For later use (Proposition 6.3) we state

Lemma 2.2. Let $Z$ be a complex manifold and $M \subset Z$ a (connected real-analytic) generic and minimal $C R$-submanifold. Then $M \backslash A$ is connected for every closed complex-analytic subset $A \subset Z$.

Proof. We first show that the proof of the Lemma can be reduced to the case when $A \subset Z$ is non-singular. Indeed, there is an integer $k \geq 1$ and a descending chain $A=A_{0} \supset \cdots \supset A_{k}=\emptyset$ of analytic subsets such that $A_{j}$ is the singular locus of $A_{j-1}$ for all $j=1, \ldots, k$. Put $M_{j}:=M \backslash A_{j}$. Then $A_{j-1} \backslash A_{j}$ is analytic in $Z_{j}:=$ $Z \backslash A_{j}$ and $M_{j-1}=M_{j} \backslash\left(A_{j-1} \backslash A_{j}\right)$. Therefore it suffices to show inductively that $M=M_{k}, M_{k-1}, \ldots, M_{0}$ all are connected. For the rest of the proof we therefore assume that $A$ is nonsingular and also, contrary to the claim of the Lemma, that $M \backslash A$ is disconnected. Notice that this implies

$$
T_{a} M \cap T_{a} A \neq T_{a} M \text { for all } a \in M \cap A,
$$

since otherwise $M \backslash A=\emptyset$ would be connected as a consequence of $T_{a} Z=T_{a} M+$ $i T_{a} M \subset T_{a} A \subset T_{a} Z$.

The intersection $S:=A \cap M$ is a real-analytic set. Again, there is an integer $r \geq 1$ and a descending chain $S=S_{0} \supset \cdots \supset S_{r}=\emptyset$ of real-analytic subsets such that $S_{j}$ is the singular locus of $S_{j-1}$ for all $j=1, \ldots, r$. Choose $j \leq r$ minimal with respect to the property that $M \backslash S_{j}$ is connected. Then $j>0$ by the above assumption and $M \backslash S_{j-1}=\left(M \backslash S_{j}\right) \backslash\left(S_{j-1} \backslash S_{j}\right)$ is disconnected. In particular, also $\left(M \backslash S_{j}\right) \backslash N$ is disconnected, where we denote by $N$ the union of all connected components of $\left(S_{j-1} \backslash S_{j}\right)$ that have codimension 1 in $M$. Clearly, (*) improves to

$$
T_{a} M \cap T_{a} A=T_{a} N \text { for all } a \in N .
$$

Since $M$ is minimal by assumption there exists an $a \in N$ with $H_{a} M \not \subset T_{a} N$ and hence with $H_{a} M \not \subset T_{a} A$ by (**). Since $H_{a} M$ and $T_{a} A$ are complex linear 
subspaces, there is a linear subspace $V \subset H_{a} M \subset T_{a} M$ of real dimension $\geq 2$ with $H_{a} M=V \oplus\left(H_{a} M \cap T_{a} A\right)$. But then $V \cap T_{a} N=V \cap\left(T_{a} M \cap T_{a} A\right)=0$ gives a contradiction since $T_{a} N$ is a real hyperplane in $T_{a} M$. This shows that $M \backslash A$ cannot be assumed to be disconnected, and the proof is complete.

Notice that the assumption on $M$ in Lemma 2.2 is automatically satisfied if $M$ is of hypersurface type and has nowhere vanishing Levi form. Indeed, if $M$ is a hypersurface and is not minimal in $a \in M$ then the Levi form of $M$ at $a$ vanishes.

Convention for notating vector fields. In this paper we do not need the complexified tangent bundle $T M \otimes_{\mathbb{R}} \mathbb{C}$ of $M$. All vector fields occurring here correspond to 'real vector fields' elsewhere. In particular, if $E$ is a complex vector space of finite dimension and $U \subset E$ is an open subset then the vector fields $\xi \in \mathfrak{h o l}(U)$ correspond to holomorphic mappings $f: U \rightarrow E$, and the correspondence is given in terms of the canonical trivialization $T U \cong U \times E$ by identifying the mapping $f$ with the vector field $\xi=\left(\operatorname{id}_{U}, f\right)$. To have a short notation we also write

$$
\xi=f(z) \partial / \partial z
$$

As soon as the vector field $\xi=f(z) \partial / \partial z$ is considered as differential operator, special caution is necessary: $\xi$ applied to the smooth function $h$ on $U$ is $\xi h=$ $f(z) \partial / \partial z h+\bar{f}(z) \partial / \partial \bar{z} h$. We therefore stress again that we write

$$
\xi=f(z) \partial / \partial z \quad \text { instead of } \quad \xi=f(z) \partial / \partial z+\bar{f}(z) \partial / \partial \bar{z} \text { elsewhere, }
$$

and this convention will be in effect allover the paper.

\section{Tube manifolds}

Throughout this section let $V$ be a real vector space of finite dimension and $E:=$ $V \oplus i V$ its complexification. For every (connected and locally closed) real-analytic submanifold $F \subset V$ the manifold

$$
T:=T_{F}:=V+i F \subset E
$$

is a CR-submanifold of $E$, called the tube over the base $F$. Obviously, a realanalytic submanifold $M \subset E$ is a tube in this sense if and only if $M+V=M$. Tubes form a very special class of CR-manifolds. For instance, Aut $(T)$ contains the following Abelian translation group isomorphic to the vector group $V$

$$
\Gamma:=\{z \mapsto z+v: v \in V\} .
$$

Since $T=\Gamma(i F)$ it is enough to study the local CR-structure of the tube $T$ only at points $i a \in i F \subset T$. For these

$$
T_{i a} T=V \oplus i T_{a} F \text { and } H_{i a} T=T_{a} F \oplus i T_{a} F \subset E
$$


is easily seen. In particular, $T$ is generic in $E$. For every further tube $T^{\prime}=V^{\prime}+i F^{\prime}$ in a complex vector space $E^{\prime}=V^{\prime} \oplus i V^{\prime}$ with $F^{\prime} \subset V^{\prime}$ every real affine mapping $g: V \rightarrow V^{\prime}$ with $g(F) \subset F^{\prime}$ extends to a complex affine mapping $\tilde{g}: E \rightarrow E^{\prime}$ with $\tilde{g}(T) \subset T^{\prime}$ and thus gives a CR-map $T \rightarrow T^{\prime}$. Therefore, $F$ (locally) being affinely homogeneous implies that the tube $T$ is (locally) CR-homogeneous. The converse is not true in general.

Lemma 3.1. Suppose that $T=V+i F$ is a tube submanifold of the complex vector space $E=V \oplus i V$ and that $a \in T$ is an arbitrary point. Then the following conditions are equivalent.

(i) $T$ is of finite type at a.

(ii) $T$ is minimal at $a$.

(iii) The smallest affine subspace of $V$ containing $F$ is $V$ itself.

Proof. It is enough to show the implication (iii) $\Longrightarrow$ (i). We therefore assume (iii) and identify $E=\mathbb{C}^{n}$ with $\mathbb{R}^{n} \times \mathbb{R}^{n}$ in the standard way via $(x+i y) \cong(x, y)$. Without loss of generality we assume that $T$ contains the origin of $E$ and is given in a suitable neighbourhood of it by real-analytic equations

$$
y_{j}=f_{j}\left(y_{1}, \ldots, y_{k}\right), \quad k<j \leq n,
$$

where every $f_{j}$ vanishes of order $\geq 2$ at the origin of $\mathbb{R}^{k}$. The assumption (iii) implies that the germs of the functions $f_{k+1}, \ldots, f_{n}$ at $0 \in \mathbb{R}^{k}$ are linearly independent. For all $1 \leq \ell, m \leq k$ the vector fields

$$
\xi^{\ell}:=\partial / \partial x_{\ell}+\sum_{j>k} \partial f_{j} / \partial y_{\ell} \partial / \partial x_{j} \quad \text { and } \quad \eta^{m}:=\partial / \partial y_{m}+\sum_{j>k} \partial f_{j} / \partial y_{m} \partial / \partial y_{j}
$$

(expressed in the real coordinates $(x, y)$ of $E$ ) are sections in the holomorphic subbundle $H T$ over the tube manifold $T$. Also, for every multi-index $v=\left(v_{1}, \ldots, v_{k}\right) \in$ $\mathbb{N}^{k}$ with $|v|:=v_{1}+\cdots+v_{k} \geq 1$ and every $\ell=1, \ldots, k$ we have

$$
\left(\operatorname{ad} \eta^{1}\right)^{\nu_{1}}\left(\operatorname{ad} \eta^{2}\right)^{\nu_{2}} \cdots\left(\operatorname{ad} \eta^{\mathrm{k}}\right)^{\nu_{\mathrm{k}}} \xi^{\ell}=\sum_{\mathrm{j}>\mathrm{k}}\left(\partial^{|\nu|} / \partial y^{\nu}\left(\partial f_{j} / \partial y_{\ell}\right)\right) \partial / \partial x_{j} .
$$

Denote by $S \subset T_{0} T$ the linear subspace spanned by $H_{0} T$ and all vector fields (3.1). Assume that there exists a non-trivial linear form $\lambda$ on $T_{0} T$ with $\lambda(S)=0$ and put $f:=\sum_{j>k} d_{j} f_{j}$ with $d_{j}:=\lambda\left(\partial / \partial x_{j}\right)$. Then $d_{j} \neq 0$ for some $j>k$, that is, $f \not \equiv 0$. On the other hand, (3.1) shows that all partial derivatives of $f$ of order $\geq 2$ vanish at the origin. By choice of the functions $f_{j}$ also all partial derivatives of $f$ of order $<2$ vanish, a contradiction. Therefore $S=T_{0} T$ and (i) must hold.

Proposition 3.2. Suppose that $T=V+i F$ is a tube submanifold of the complex vector space $E=V \oplus i V$ and suppose, without loss of generality, that $T$ contains the origin of E. Then there exist complex linear subspaces $E^{\prime}, E^{\prime \prime}$ of $E$ and tube submanifolds $T^{\prime} \subset E^{\prime}, T^{\prime \prime} \subset E^{\prime \prime}$ with the following properties: 
(i) $T^{\prime}$ is an $\mathbb{R}$-linear subspace of $E^{\prime}$ with $E^{\prime}=T^{\prime}+i T^{\prime}$.

(ii) $T^{\prime \prime}$ is holomorphically nondegenerate and of finite type at every point.

(iii) $E=E^{\prime} \oplus E^{\prime \prime}$ and $T$ is open in $T^{\prime}+T^{\prime \prime}$.

Proof. By Lemma 3.1 we assume without loss of generality that $V$ is the linear span of $F$. We then verify the claim with $T^{\prime}=E^{\prime}$.

For every $a \in T$ put $\mathfrak{h}_{a}:=\mathfrak{h o l}(T, a) \cap i \mathfrak{h o l}(T, a)$ and $E_{a}:=\varepsilon_{a}\left(\mathfrak{h}_{a}\right)$. Then $\mathfrak{h}_{a}$ is a complex Lie subalgebra of $\mathfrak{h o l}(E, a)$ and $E_{a} \subset E$ is a complex linear subspace. Denote by $M \subset T$ the subset of all points at which the function $a \mapsto \operatorname{dim} E_{a}$ takes a global maximum and fix a connected component $S$ of $M$. Then $S$ is open in $T$ and $k:=\operatorname{dim} E_{a}$ does not depend on $a \in S$. Let $\mathbb{G}$ be the Grassmannian of all $k$-planes in $E$ and consider the map $\phi: S \rightarrow \mathbb{G}, a \mapsto E_{a}$. For every $a \in S$ the map $\phi$ is constant on $(a+V) \subset S$. Since $\phi$ is $\mathrm{CR}$ we conclude that $T^{\prime}:=E^{\prime}:=E_{a}$ does not depend on $a \in S$. Now fix an arbitrary vector $\alpha \in E^{\prime}$ and consider the constant vector field $\xi=\alpha \partial / \partial z$ on $E$. Since $\xi$ is tangent to $S$ it is also tangent to $T$, that is, the germ $\xi_{a} \in \mathfrak{h o l}(E, a)$ is contained in $\mathfrak{h}_{a}$ for all $a \in T$. As a consequence we get $E^{\prime} \subset E_{a}$ and thus $E^{\prime}=E_{a}$ for all $a \in T$. There exists a linear subspace $V^{\prime \prime} \subset V$ with $E=E^{\prime} \oplus E^{\prime \prime}$ for $E^{\prime \prime}:=V^{\prime \prime} \oplus i V^{\prime \prime}$. The image $T^{\prime \prime}$ of $T$ with respect to the canonical projection $E \rightarrow E^{\prime \prime}$ is a tube submanifold of $E^{\prime \prime}$ satisfying (iii). The base $F^{\prime \prime}$ of $T^{\prime \prime}$ spans the vector space $V^{\prime \prime}$, that is, $T^{\prime \prime}$ is of finite type by Lemma 3.1 . For the proof of the first part in (ii) we may assume without loss of generality that $E^{\prime}=0$ holds, that is, $E=E^{\prime \prime}$. But then by the above arguments we have $\mathfrak{h}_{a}=0$ for all $a \in T$, that is, $T=T^{\prime \prime}$ is holomorphically nondegenerate.

It is known that for every holomorphically nondegenerate minimal CR-manifold germ $(M, a)$ the Lie algebra $\mathfrak{h o l}(M, a)$ has finite dimension, $c f$. in [3, Theorem 12.5.3]. Calling a CR-manifold germ $(M, a)$ of tube type if it is CR-isomorphic to a germ $(T, c)$ with $T$ a tube manifold we therefore get the

Corollary 3.3. Let $(M, a)$ be a CR-manifold germ of tube type. Then there exist unique integers $k, l \geq 0$ and a holomorphically nondegenerate CR-submanifold $M^{\prime} \subset M$ of finite type with $a \in M^{\prime}$ such that $(M, a)$ is $C R$-isomorphic to the direct product $\left(\mathbb{C}^{k}, 0\right) \times\left(\mathbb{R}^{l}, 0\right) \times\left(M^{\prime}, a\right)$. Furthermore:

(i) $(M, a)$ is holomorphically nondegenerate if and only if $k=0$.

(ii) $(M, a)$ is of finite type if and only if $l=0$.

(iii) $\mathfrak{h o l}(M, a)$ has finite dimension if and only if $k=l=0$.

As shown in [4], to every real-analytic CR-submanifold $M \subset \mathbb{C}^{n}$ there exists a proper real-analytic subset $A \subset M$ such that the germ $(M, a)$ is CR-isomorphic to $\left(\mathbb{C}^{k}, 0\right) \times\left(M^{\prime}, a\right)$ for some $k \geq 0$ and some holomorphically nondegenerate CRsubmanifold $M^{\prime} \subset M$ containing $a$, provided $a \in M \backslash A$. Corollary 3.3 implies that $A$ can be chosen to be empty if $M$ is of tube type.

An analyticity criterion. In the following $k$-differentiable always means $\mathcal{C}^{k}$ for $1 \leq k \leq \infty$. For every abstract $k$-differentiable CR-manifold $N$ then the tangent bundle $T N$ is of class $\mathcal{C}^{k-1}$ and we denote by $\mathfrak{X}^{k-1}(N)$ the $\mathbb{R}$-linear space of 
$(k-1)$-differentiable infinitesimal CR-transformations on $N$. Unless $k=k-1=$ $\infty$, the space $\mathfrak{X}^{k-1}(N)$ is not a Lie algebra in general. But again, for every $k$ differentiable CR-diffeomorphism $\phi: N \rightarrow M$ we have a canonical linear isomorphism $\phi_{*}: \mathfrak{X}^{k-1}(N) \rightarrow \mathfrak{X}^{k-1}(M)$. Clearly, every real-analytic CR-manifold $M$ can be considered as a $k$-differentiable CR-manifold in a canonical way and $\mathfrak{h o l}(M) \subset \mathfrak{X}^{k-1}(M)$ in this sense.

Proposition 3.4. Let $M$ be a real-analytic holomorphically nondegenerate $C R$ manifold and let $V+i F$ be a $k$-differentiable tube submanifold of the complex vector space $E:=V \oplus i V$. Suppose that $N$ is an open subset of $V+i F$ and that there exists a $k$-differentiable CR-diffeomorphism $\phi: N \rightarrow M$ with $\phi_{\mathfrak{*}} \mathfrak{v} \subset \mathfrak{h o l}(M)$ for $\mathfrak{v}:=\{v \partial / \partial z: v \in V\} \subset \mathfrak{X}^{k-1}(N)$. Then $N \subset E$ is a (locally-closed) real-analytic subset of $E$ and $\phi$ is a bianalytic CR-diffeomorphism.

Proof. Fix an arbitrary point $a \in M$. Since the claim is of local nature we may assume that $M$ is generically embedded in $E$. The local flows of vector fields in $\mathfrak{v}$ commute. Therefore the image $\mathfrak{w}:=\phi_{*} \mathfrak{b}$ is an Abelian subalgebra of $\mathfrak{h o l}(M) \subset$ $\mathfrak{h o l}(M, a)$ and $\varepsilon_{a}\left(\mathfrak{w}^{\mathbb{C}}\right)=E$. By Proposition 4.1 we may assume without loss of generality that $M=V+i H$ is a real-analytic tube submanifold of $E$ and that $\mathfrak{w}=\mathfrak{v} \subset \mathfrak{h o l}(M, a)$. Applying a suitable affine transformation to $M$ we may assume in addition that $a \in N, \phi(a)=a$ and $\phi: \mathfrak{v} \rightarrow \mathfrak{v}$ is the identity. For suitable open subsets $U, W \subset V$ we may assume furthermore that $F \subset W, N=$ $U+i F$ and that there exist $k$-differentiable functions $f, g: U \times W \rightarrow V$ satisfying $\phi(z)=f(x, y)+i g(x, y)$ for all $x, y \in U$ with $z=x+i y \in N$. The condition $\phi_{*}=\operatorname{id}_{\mathfrak{v}}$ implies $\partial f / \partial x \equiv \mathrm{id}_{V}$ and $\partial g / \partial x \equiv 0$ on $U \times F$. The CR-property then gives $\partial f /\left.\partial y\right|_{c}(v)=0$ and $\partial g /\left.\partial y\right|_{c}(v)=v$ for all $c=(e, f) \in U \times F$ and $v \in T_{f} F$. Because of $\phi(a)=a$ this implies $\phi(z)=z$ for all $z \in N$ near $a$, that is, the manifold germs $(N, a)$ and $(M, a)$ coincide.

Proposition 3.4 implies that in case $\mathfrak{X}^{k-1}(M, a)=\mathfrak{h o l}(M, a)$ for every $a \in M$, every $k$-differentiable tube realization $N \subset E$ of $M$ is real-analytic. This happens, for instance with $k=1$, if $M$ is of hypersurface type with nowhere vanishing Levi form. Indeed, by Theorem 3.1 in [2] every 1-differentiable CR-diffeomorphism between open subsets of $M$ is real-analytic.

\section{Tube realizations}

In the following $M$ is a CR-manifold generically embedded in the complex manifold $Z$ and $a \in M$ is a given point. Then the tube realizations $\phi:(M, a) \rightarrow(T, c)$ and $\phi:(M, a) \rightarrow\left(T^{\prime}, c^{\prime}\right)$ with tubes $T \subset E, T^{\prime} \subset E^{\prime}$ as in Section 3 are called affinely equivalent if the tube germs $(T, c),\left(T^{\prime}, c^{\prime}\right)$ are equivalent under an affine isomorphism $\lambda: E \rightarrow E^{\prime}$, or equivalently, if $\phi^{\prime} \circ g=\lambda \circ \phi$ for some $g \in \operatorname{Aut}(M, a)$ and some affine isomorphism $\lambda$. Also, we call the subsets $\mathfrak{v}, \mathfrak{v}^{\prime} \subset \mathfrak{h o l}(M, a)$ conjugate with respect to $\operatorname{Aut}(M, a)$ if $\mathfrak{v}^{\prime}=g_{*}(\mathfrak{v})$ for some $g \in \operatorname{Aut}(M, a)$. 
Proposition 4.1. The affine equivalence classes of tube realizations of the germ $(M, a)$ are in 1-1-correspondence to the Aut $(M, a)$-conjugacy classes of Abelian Lie subalgebras $\mathfrak{v} \subset \mathfrak{g}:=\mathfrak{h o l}(M, a)$ satisfying

(i) $\mathfrak{v}$ is totally real in $\mathfrak{h o l}(Z, a)$,

(ii) $\mathfrak{e}:=\mathfrak{v} \oplus$ iv $\subset \mathfrak{h o l}(Z, a)$ spans the full tangent space $T_{a} Z$.

Proof. Suppose that for the tube submanifold $T=V+i F \subset E$ the CR-isomorphism $\phi:(M, a) \rightarrow(T, c)$ is given. Then $\mathfrak{v}:=\left\{\phi^{*}(v \partial / \partial z): v \in V\right\} \subset \mathfrak{g}$ satisfies the conditions (i) and (ii). For every affine isomorphism $\lambda: E \rightarrow E^{\prime}$ and every tube realization $\phi^{\prime}:(M, a) \rightarrow\left(T^{\prime}, c^{\prime}\right):=\lambda(T, c)$ the transformation $g:=\phi^{\prime-1} \circ \lambda \circ \phi \in$ Aut $(M, a)$ satisfies $g_{*}(\mathfrak{v})=\left\{\phi^{\prime *}\left(v^{\prime} \partial / \partial z\right): v^{\prime} \in V^{\prime}\right\}$ for $V^{\prime}:=\lambda(V)$.

Conversely, suppose that an Abelian Lie subalgebra $\mathfrak{v} \subset \mathfrak{g}$ with (i), (ii) is given. Then $\mathfrak{e}$ is an Abelian complex Lie algebra and by Lemma 2.1 the evaluation map $\varepsilon_{a}: \mathfrak{e} \rightarrow T_{a} Z$ is a complex linear isomorphism. Denote by $E$ the complex vector space underlying $\mathfrak{e}$ and by $V \subset E$ the real vector space underlying $\mathfrak{v}$. By the implicit function theorem there exist open neighbourhoods $U$ of $0 \in E$ and $W$ of $a \in Z$ such that $\psi(\xi):=\exp (\xi)(a) \in W$ is defined for every vector field $\xi \in U$ and $\psi: U \rightarrow W$ is a biholomorphic mapping with $\psi(0)=a$. For $\phi:=\psi^{-1}$ then $\phi(W \cap M)$ is an open piece of a tube $T=V+i F \subset E$, that is, $\phi:(M, a) \rightarrow(T, 0)$ gives a tube realization with $\mathfrak{v}=\left\{\phi^{*}(v \partial / \partial z): v \in V\right\}$. Now fix a $g \in \operatorname{Aut}(M, a)$. Then also $\mathfrak{v}^{\prime}:=g_{*}(\mathfrak{v})$ with $\mathfrak{e}^{\prime}:=\mathfrak{v}^{\prime} \oplus i \mathfrak{v}^{\prime}$ satisfies (i), (ii) and thus gives a tube realization $\phi^{\prime}:(M, a) \rightarrow\left(T^{\prime}, 0\right)$ according to the procedure above. Since $\mathfrak{e}, \mathfrak{e}^{\prime}$ are Abelian, there is a complex linear isomorphism $\lambda: E \rightarrow E^{\prime}$ with $\lambda(\psi(\xi))=$ $\psi^{\prime}\left(g_{*}(\xi)\right)$ for all $\xi$ in a neighbourhood of the origin in $E$. But this means that $\lambda:(T, 0) \rightarrow\left(T^{\prime}, 0\right)$ is an affine equivalence.

Notice that $\mathfrak{e}$ is maximal Abelian in $\mathfrak{h o l}(Z, a)$ by Lemma 2.1. In case $M$ is holomorphically non-degenerate the condition (i) above is automatically satisfied and $\mathfrak{v}$ is maximal Abelian in $\mathfrak{h} \mathfrak{o l}(M, a)$.

Remark 4.2. A different characterization of Abelian Lie subalgebras $\mathfrak{v}$ giving rise to tube realizations of $(M, a)$ occurs already in [1]. Instead of (i), (ii) there $\mathfrak{v}$ has to act without isotropy and transversally to the holomorphic tangent bundle.

Tubes $T=V+i F$ have a special property: $\tau(x+i y):=-x+i y$ for all $x \in V, y \in F$ defines an anti-CR map $\tau: T \rightarrow T$ with $\tau^{2}=$ id and $\tau(a)=a$ for all $a \in i F \subset T$. This motivates the following considerations.

Involutions. In this subsection $M$ stands for an arbitrary CR-manifold. A realanalytic mapping $\tau: M \rightarrow M$ is called an involution of $M$ if it is anti-CR and satisfies $\tau^{2}=\mathrm{id}$. If in addition $\tau(a)=a$ for a given $a \in M$ we call $\tau$ an involution of $M$ at $a$ or of the CR-manifold germ $(M, a)$. Two involutions $\tau, \tau^{\prime}$ of $(M, a)$ are called equivalent if $\tau^{\prime}=g \tau g^{-1}$ for some $g \in$ Aut $(M, a)$. Every involution $\tau$ of $(M, a)$ splits various linear spaces, associated with the germ $(M, a)$, into their \pm 1 -eigenspaces. To indicate the dependence on $\tau$ we mark the +1 -eigenspaces by an upper index $\tau$ and the -1 -eigenspaces by an upper index $-\tau, e . g . T_{a} M=$ 
$T_{a}^{\tau} M \oplus T_{a}^{-\tau} M, H_{a} M=H_{a}^{\tau} M \oplus H_{a}^{-\tau} M$ and $\mathfrak{g}=\mathfrak{g}^{\tau} \oplus \mathfrak{g}^{-\tau}$ for $\mathfrak{g}:=\mathfrak{h o l}(M, a)$. Clearly $\left(T_{a} M\right)^{\tau}=T_{a}\left(M^{\tau}\right)$. Crucial for the explicit determination of all tube realizations for $(M, a)$ is the invariant

$$
\vartheta_{a}(\tau):=\operatorname{dim}\left(T_{a} M / H_{a} M\right)^{\tau}
$$

and the following reformulation of Proposition 4.1.

Proposition 4.3. Proposition 4.1 remains valid if (i) is replaced by

(i') There exists an involution $\tau$ of $(M, a)$ with $\mathfrak{v} \subset \mathfrak{g}^{-\tau}$.

For every $\mathfrak{v} \subset \mathfrak{g}$ with (i), (ii) the involution $\tau$ in (i') is uniquely determined and satisfies $\vartheta_{a}(\tau)=0$. In particular, for every $g \in \operatorname{Aut}(M, a)$ the involution $\tau^{\prime}$ corresponding to $\mathfrak{v}^{\prime}:=g_{*}(\mathfrak{v}) \subset \mathfrak{g}$ is given by $\tau^{\prime}=g \circ \tau \circ g^{-1}$.

Proof. (i') $\Longrightarrow$ (i) is obvious. Therefore let us assume conversely that the Abelian subalgebra $\mathfrak{v} \subset \mathfrak{g}$ satisfies (i) and (ii). Without loss of generality we assume by Proposition 4.1 that $M=V+i F$ is a tube submanifold of $E=V \oplus i V$, that $a \in i F$ and that $\mathfrak{v}=\{v \partial / \partial z: v \in V\}$. Then the involution $\tau(x+i y)=-x+i y$ of $(M, a)$ satisfies (i') and $\vartheta_{a}(\tau)=0$. Now suppose that $\tau^{\prime}$ is a further involution of $(M, a)$ with the same properties. Then $g:=\tau \circ \tau^{\prime} \in \operatorname{Aut}(M, a)$ satisfies $g_{*}(\alpha \partial / \partial z)=$ $\alpha \partial / \partial z$ for all $\alpha \in V$ and hence also for all $\alpha \in E$. But then $g=$ id and $\tau^{\prime}=\tau$.

Remark 4.4. The explicit determination of all tube realizations for $(M, a)$ up to affine equivalence requires by Proposition 4.1 that, up to conjugation by the stability group Aut $(M, a)$, all Abelian Lie subalgebras $\mathfrak{v} \subset \mathfrak{h o r}(M, a)$ have to be found that satisfy the conditions (i), (ii). Proposition 4.3 restricts the search (and with it the amount of computation) to the following: Determine first, up to conjugation, all involutions of $(M, a)$ that satisfy $\vartheta_{a}(\tau)=0$ and then, for every such involution $\tau$, search for suitable $\mathfrak{v}$ 's in $\mathfrak{g}^{-\tau}$. As an application of that method we classify algebraically in the forthcoming paper [11] all local tube realizations of Levi nondegenerate hyperquadrics $Q \subset \mathbb{C}^{n}$. These are locally CR-equivalent to the hypersurfaces $S_{p, q}:=S_{p, q}^{1}$ occurring in the next section and have the special property that every germ $(Q, a)$ has, up to equivalence, a unique involution satisfying $\vartheta_{a}(\tau)=0$.

\section{Classification of involutions for certain CR-manifolds}

Fix in the following arbitrary integers $p, q \geq m \geq 1$ and denote by $\mathbb{G}$ the Grassmannian of all linear $m$-spaces in $\mathbb{C}^{n}, n:=p+q$. Then $\mathbb{G}$ is a compact complex manifold of dimension $m(n-m)$ on which $\operatorname{SL}(n, \mathbb{C})$ acts transitively by holomorphic transformations. The group Aut $(\mathbb{G})$ coincides with $\operatorname{PSL}(n, \mathbb{C})=$ $\mathrm{SL}(n, \mathbb{C}) /$ center, unless $p=q>1$ (in which case there is a second connected component of Aut $(\mathbb{G})$ ). To avoid totally real examples we exclude the case $p=q=m$ for the rest of the section. 
Consider on $\mathbb{C}^{n}$ the real-valued function $h$ defined by

$$
h(z)=(u \mid u)-(v \mid v) \text { for all } \quad z=(u, v) \in \mathbb{C}^{p} \oplus \mathbb{C}^{q}
$$

with ( $\mid$ ) being the standard inner product and identify $\operatorname{SU}(p, q) \subset \operatorname{SL}(n, \mathbb{C})$ with the subgroup of all transformations leaving $h$ invariant. Then the connected real submanifold

$$
S:=S_{p, q}^{m}:=\{L \in \mathbb{G}: h(L)=0\}
$$

is the unique closed (and hence compact) $\mathrm{SU}(p, q)$-orbit in $\mathbb{G}$. As CR-submanifold $S$ is generically embedded in $\mathbb{G}$ with CR-dimension $m(n-2 m)$ and CRcodimension $m^{2}$. Furthermore, a dense open subset of $S$ can be realized as a real quadric in $\mathbb{C}^{m(n-m)}$, and $\mathfrak{g}:=\mathfrak{s u}(p, q)=\mathfrak{h o l}(S) \cong \mathfrak{h o l}(S, a)$ holds for every $a \in S, c f$. [17] for details. As a consequence of Theorem 1.3 in [15], $c f$. also [8], every CR-isomorphism between domains $D_{1}, D_{2}$ of $S$ extends to a biholomorphic automorphism of $\mathbb{G}$ leaving $S$ invariant. Since $S$ has a global (anti-CR) involution (see the following classification) also every anti-CR-isomorphism between domains $D_{1}, D_{2}$ of $S$ extends to a global antiholomorphic automorphism of $\mathbb{G}$ leaving $S$ invariant. For the classification of all involutions of the germ $(S, a)$ it is therefore enough to determine all global involutions of $S$.

Classification of all involutions on S. Let a global involution $\tau$ of $S$ (not necessarily having a fixed point) be given. Then $\tau$ extends to an antiholomorphic automorphism of $\mathbb{G}$ that we also denote by $\tau$. Also, the involution induced by $\tau$ on $\mathfrak{l}:=\mathfrak{s l}(n, \mathbb{C}) \cong \mathfrak{a u t}(\mathbb{G})$ will be denoted by the same symbol. The fixed point submanifold $\mathbb{G}^{\tau}$ of $\mathbb{G}$ is either empty or a real form of $\mathbb{G}$. One can show that there are integers $\varepsilon, \delta$ with $\varepsilon^{2}=\delta^{2}=1$ together with an antilinear endomorphism $\tilde{\tau}$ of $\mathbb{C}^{n}$ such that $\tilde{\tau}^{2}=\varepsilon$ id, $h \circ \tilde{\tau}=\delta h$ and $\tau(L)=\{\tilde{\tau}(z): z \in L\}$ for all $L \in \mathbb{G}$. Depending on the value of $\varepsilon$ we have the following two cases.

$\varepsilon=1:$ Then $\mathfrak{l}^{\tau} \cong \mathfrak{s l}(n, \mathbb{R})$ and $\mathbb{G}^{\tau}$ can be identified with the real Grassmannian of all real linear m-spaces in $\mathbb{R}^{n}$.

$\varepsilon=-1$ : This case can only occur if $n$ is even and then $\mathfrak{l}^{\tau} \cong \mathfrak{s l}(n / 2, \mathbb{H})$, where $\mathbb{H}$ is the field of quaternions. Furthermore, $\mathbb{G}^{\tau}$ is empty if and only if $m$ is odd.

The precise classification requires some work. Here we state only the final result: It turns out that for every given $p, q$ the possible pairs $(\varepsilon, \delta)$ stand in a one-to-one relation with the $\mathrm{SU}(p, q)$-conjugation classes of involutions on $S=S_{p, q}^{m}$. More explicitly, every such involution is conjugate to exactly one of the following four types $\mathbf{I}-\mathbf{I V}$, where we write every (row) $z \in \mathbb{C}^{n}$ in the form $z=(u, v)$ with $u \in \mathbb{C}^{p}$ 
and $v \in \mathbb{C}^{q}$. Also, for every integer $d \geq 1$ we put $J_{d}:=\left(\begin{array}{cc}0 & \mathbb{1} \\ -11 & 0\end{array}\right) \in \operatorname{GL}(2 \mathrm{~d}, \mathbb{Z})$ :

\begin{tabular}{|c|c|c|c|c|c|}
\hline type & $(\varepsilon, \delta)$ & & $\tilde{\tau}(u, v)$ & $\mathfrak{l}^{\tau}$ & $\mathfrak{g}^{\tau}$ \\
\hline I & $(+1,+1)$ & & $(\bar{u}, \bar{v})$ & $\mathfrak{s l}(p+q, \mathbb{R})$ & $\mathfrak{s o}(p, q)$ \\
\hline II & $(+1,-1)$ & $p=q$ & $(\bar{v}, \bar{u})$ & $\mathfrak{s l}(2 p, \mathbb{R})$ & $\mathfrak{s p}(p, \mathbb{R})$ \\
\hline III & $(-1,+1)$ & $\begin{array}{l}p=2 p^{\prime}, \\
q=2 q^{\prime}\end{array}$ & $\left(\bar{u} J_{p^{\prime}}, \bar{v} J_{q^{\prime}}\right)$ & $\mathfrak{s l}\left(p^{\prime}+q^{\prime}, \mathbb{H}\right)$ & $\mathfrak{s p}\left(p^{\prime}, q^{\prime}\right)$ \\
\hline IV & $(-1,-1)$ & $p=q$ & $(-\bar{v}, \bar{u})$ & $\mathfrak{s l}(p, \mathbb{H})$ & $\mathfrak{s o}(p, \mathbb{H})$ \\
\hline
\end{tabular}

$S^{\tau}$ is not empty if and only if $\varepsilon=1$ or $m$ is even. In this case $\vartheta_{a}(\tau)=m(m-\delta) / 2$ holds for all $a \in S^{\tau}$. Recall that the involution $\tau$ is associated to a tube realization of $(S, a)$ only if the invariant $\vartheta_{a}(\tau)$ vanishes, see 4.3 , that is, only for $m=1$ in type I. On the other hand it is well known that every hypersurface $S_{p, q}:=S_{p, q}^{1}$ has many local tube realizations, compare $e . g$. with $[11,12]$.

A word to the computation of $\vartheta_{a}(\tau)$ above: We identify $\mathfrak{g}=\mathfrak{s u}(p, q)$ and the Lie subalgebra of all matrices in $\mathfrak{s l}(n, \mathbb{C})$ of the form $\left(\begin{array}{ll}u & v^{*} \\ v & w\end{array}\right)$ with $u=-u^{*} \in \mathbb{C}^{p \times p}$, $w=-w^{*} \in \mathbb{C}^{q \times q}$ and $v \in \mathbb{C}^{q \times p}$. The blocks are written themselves as block matrices

$$
u=\left(\begin{array}{ll}
u_{11} & u_{12} \\
u_{21} & u_{22}
\end{array}\right), \quad v=\left(\begin{array}{ll}
v_{11} & v_{12} \\
v_{21} & v_{22}
\end{array}\right), \quad w=\left(\begin{array}{ll}
w_{11} & w_{12} \\
w_{21} & w_{22}
\end{array}\right)
$$

whose sizes are determined by the requirement $u_{11}, v_{11}, w_{11} \in \mathbb{C}^{m \times m}$. Denote by $e_{1}, \ldots, e_{n}$ the standard basis of $\mathbb{C}^{n}$ and by $a \in \mathbb{G}$ the linear subspace of $\mathbb{C}^{n}$ spanned by all vectors $e_{k}+e_{p+k}$ with $1 \leq k \leq m$. Then $a$ is a point of $S=S_{p, q}^{m}$ and the isotropy subalgebra $\mathfrak{g}_{a}$ of $\mathfrak{g}$ at $a$ is given by the equations

$$
u_{11}+\left(v_{11}-v_{11}^{*}\right)-w_{11}=0, \quad u_{12}+v_{12}=0 \text { and } w_{12}+v_{21}^{*}=0 .
$$

Clearly, the tangent space $T_{a} S$ is canonically isomorphic to $\mathfrak{g} / \mathfrak{g}_{a}$. Now embed the unitary Lie algebra $\mathfrak{u}(m)$ into $\mathfrak{g}$ by identifying every $x \in \mathfrak{u}(m)$ with the matrix in $\mathfrak{g}$ having vanishing little blocks except $u_{11}=x$ and $w_{11}=-x$. Then it is not difficult to see that $\mathfrak{u}(m) \subset \mathfrak{g}$ can be canonically identified with the quotient space $T_{a} S / H_{a} S$. Furthermore, every involution $\tau$ of $(S, a)$ induces an involution on $\mathfrak{g}$ and, if $\mathfrak{u}(m) \subset \mathfrak{g}$ is $\tau$-invariant, then $\vartheta_{a}(\tau)=\operatorname{dim} \mathfrak{u}(m)^{\tau}$. For the types $\mathbf{I}$ and II above we have $a \in S^{\tau}$ and $\tau$ on $\mathfrak{u}(m)$ is given by $x \mapsto \delta \bar{x}$. For the remaining types III, IV and $m=2 m^{\prime}$ even we have to modify the involutions $\tau$ given above in order to obtain $a \in S^{\tau}$. For type III with $p, q$ even we write $\mathbb{C}^{n}$ as direct product $\mathbb{C}^{m} \times \mathbb{C}^{p-m} \times \mathbb{C}^{m} \times \mathbb{C}^{q-m}$ and define the modified $\tilde{\tau}$ on each factor $\mathbb{C}^{2 k}$ by $y \mapsto \bar{y} J_{k}$. For type IV with $p=q$ define $\tilde{\tau}$ on $\mathbb{C}^{m} \times \mathbb{C}^{p-m} \times \mathbb{C}^{m} \times \mathbb{C}^{p-m}$ by $(y, r, z, s) \mapsto\left(\bar{z} J_{m^{\prime}},-\bar{s}, \bar{y} J_{m^{\prime}}, \bar{r}\right)$. Then in both cases $\tau$ is given on $\mathfrak{u}(m)$ by $x \mapsto \delta J_{m} \bar{x} J_{m}^{-1}$. 


\section{A coarser equivalence relation}

In general, for a given tube submanifold $T=V+i F$ of $E=V+i V$, there is an infinite subset $A \subset T$ such that for every $a \neq b$ in $A$ the germs $(T, a)$, $(T, b)$ are affinely inequivalent - even if $T$ is locally homogeneous and hence all $(T, a),(T, b)$ are CR-equivalent. As an example consider in $\mathbb{C}^{2}$ the closed tube hypersurface $T=\mathbb{R}^{2}+i F$ with

$$
F:=\left\{x \in \mathbb{R}^{2}: \cos x_{1}=e^{x_{2}},\left|x_{1}\right|<\pi / 2\right\}
$$

(the boundary of the middle gray domain in Figure 8.1, Section 8). Consider the function $f(z):=\operatorname{Im}\left(z_{2}\right)$ on $T$. Then for every $a, b \in T$ the germs $(T, a),(T, b)$ are CR-isomorphic, (in fact, $T$ is locally CR-isomorphic to the Euclidean sphere $S^{3} \subset$ $\mathbb{C}^{2}$ ) but they are affinely equivalent if and only if $f(a)=f(b)$. Therefore $T$ gives rise to a continuum of mutually affinely inequivalent tube realizations of the CRgerm $(T, 0)$. This phenomenon motivates the introduction of a coarser equivalence relation that puts all germs $(T, a), a \in T$, into a single equivalence class. The construction is motivated by the concept of a sheaf:

For fixed $E=V^{\mathbb{C}}$ let $\mathcal{T}=\mathcal{T}(V)$ be the set of all (real-analytic) germs $(T, a)$ with $T=V+i F$ an arbitrary tube submanifold of $E$ and $a \in T$. Furthermore define $\pi: \mathcal{T} \rightarrow E$ by $(T, a) \mapsto a$. Then $\mathcal{T}$ becomes in the standard way a Hausdorff topological space over $E$ - the topology on $\mathcal{T}$ is the coarsest one such that for every tube submanifold $T \subset E$ the subset $[T]:=\{(T, a): a \in T\}$ is open in $\mathcal{T}$. The space $\mathcal{T}$ has in a unique way the structure of a (disconnected) CR-manifold by requiring that $\pi:[T] \rightarrow T$ is a CR-isomorphism for every tube submanifold $T \subset E$. Every real affine transformation $g \in \operatorname{Aff}(V) \subset \operatorname{Aff}(E)$ (the respective affine transformation groups) gives rise to a $\mathrm{CR}$-automorphism of $\mathcal{T}$ by $g(T, a):=(g T, g a)$, that we also denote by $g$. However, it should be noticed that the corresponding action of the Lie group $\operatorname{Aff}(V)$ on $\mathcal{T}$ is discontinuous. Nevertheless, every connected component of $\mathcal{T}$ is invariant under the (continuous) action of the translation subgroup $V \subset \operatorname{Aff}(V)$ and therefore may be considered as a generalized tube manifold over $E$. For every (connected) tube submanifold $T \subset E$ denote by $\widetilde{T}$ the connected component of $\mathcal{T}$ containing $[T]$ and call the pair $(\widetilde{T}, \pi)$ the abstract globalization of $T$ and also of every tube germ $(T, a), a \in T$. Since the translation group $V \subset \operatorname{Aff}(E)$ acts on $\widetilde{T}$ by CR-transformations we may consider $\widetilde{T}$ as tube manifold over $E$ via $\pi$.

Definition 6.1. The tube manifold germs $(T, a),\left(T^{\prime}, a^{\prime}\right)$ in $E=V^{\mathbb{C}}$ are called globally affinely equivalent if $\widetilde{T}^{\prime}=g(\widetilde{T})$ for the corresponding abstract globalizations and a suitable $g \in \operatorname{Aff}(V)$.

In case $\pi(\widetilde{T})$ is a (locally closed) submanifold of $E$, we call $\pi(\widetilde{T})$ the globalization of $(T, a)$ and denote it by $\hat{T}$. Clearly, then $\pi: \widetilde{T} \rightarrow \hat{T}$ is a CR-isomorphism. Furthermore, $\hat{T}$ is a tube submanifold of $E$ containing $T$ as an open submanifold and also is maximal with respect to this property. As an example, every closed tube submanifold $T \subset E$ is the globalization of each of its germs $(T, a), a \in T$. 
In the following we assume for the CR-manifold germ $(M, a)$ that the Lie algebra $\mathfrak{g}:=\mathfrak{h o l}(M, a)$ has finite dimension. Then, in particular, $(M, a)$ is holomorphically nondegenerate and we denote as usual with Int $(\mathfrak{g}) \subset$ Aut $(\mathfrak{g})$ the inner automorphism group of $\mathfrak{g}$, that is, the subgroup generated by all $\exp (\operatorname{ad} \xi), \xi \in \mathfrak{g}$. Finally, for every $a \in M$ let

$$
\rho_{a}: \mathfrak{h o l}(M) \hookrightarrow \operatorname{hol}(M, a) \quad \text { be the restriction mapping. Then we have }
$$

Lemma 6.2. Suppose that $\rho_{a}: \mathfrak{h o l}(M) \rightarrow \mathfrak{g}=\mathfrak{h o l}(M, a)$ is bijective. Then $g \mapsto \rho_{a} g_{*} \rho_{a}^{-1}$ defines a group homomorphism Aut $(M) \rightarrow$ Aut $(\mathfrak{g})$ that sends $H$ to Int $(\mathfrak{g})$, where $H \subset$ Aut $(M)$ denotes the subgroup generated by $\exp (\mathfrak{a u t}(M))$. For every $g \in$ Aut $(M)$ and $b:=g(a)$ also $\rho_{b}: \mathfrak{h o l}(M) \rightarrow \mathfrak{h o l}(M, b)$ is bijective. Furthermore, for every Abelian subalgebra $\mathfrak{w} \subset \mathfrak{h o l}(M)$ such that $\rho_{a}(\mathfrak{w}) \subset \mathfrak{g}$ gives a local tube realization, also $\rho_{b}\left(g_{*} \mathfrak{w}\right) \subset \mathfrak{h o l}(M, b)$ gives a local tube realization and both are affinely equivalent.

Proof. From $\rho_{a} \operatorname{Ad}(\exp \xi)=\rho_{\mathrm{a}} \exp (\operatorname{ad} \xi)=\exp \left(\operatorname{ad} \rho_{\mathrm{a}}(\xi)\right) \rho_{\mathrm{a}}$ for all $\xi \in \mathfrak{a u t}(M)$ we see that $H$ maps into Int ( $\mathfrak{g})$. The other statements are obvious.

The following global statement will be one of the key ingredients in the proof of the following Theorem 7.1. Both of these results allow to reduce the classification problem for tube realizations of $(M, a)$ in many cases to a purely algebraic one.

Proposition 6.3. Let $Z$ be a complex manifold and $M \subset Z$ a generically embedded minimal CR-submanifold. Assume that, for a given point $a \in M, \mathfrak{g}:=\mathfrak{h o l}(M, a)$ has finite dimension and every germ in $\mathfrak{g}$ extends to a vector field in $\mathfrak{a u t}(M)$. Let $\mathfrak{v}, \mathfrak{v}^{\prime} \subset \mathfrak{g}$ be Abelian subalgebras giving rise to local tube realizations of $(M, a)$ according to Proposition 4.1 and assume that every germ in $\mathfrak{e}:=\mathfrak{v}^{\mathbb{C}} \subset \mathfrak{h o l}(Z, a)$ extends to a vector field in $\mathfrak{a u t}(Z)$. Then the local tube realizations of $(M, a)$ given by $\mathfrak{v}, \mathfrak{v}^{\prime}$ are globally affinely equivalent if $\mathfrak{v}=\lambda\left(\mathfrak{v}^{\prime}\right)$ for some $\lambda \in \operatorname{Int}(\mathfrak{g})$.

Proof. For simpler notation we identify the Lie algebras $\mathfrak{h o l}(M)$ and $\mathfrak{g}$ via the isomorphism $\rho_{a}: \mathfrak{h o l}(M) \rightarrow \mathfrak{g}$. Since $\mathfrak{a u t}(M)=\mathfrak{h o l}(M)$, for every $\lambda \in \operatorname{Int}(\mathfrak{g})$ with $\mathfrak{v}=\lambda\left(\mathfrak{v}^{\prime}\right)$ there exists a transformation $g \in G$ with $\lambda=\operatorname{Ad}(\mathrm{g})=\mathrm{g}_{*}$, where $G$ is the group $H$ from Lemma 6.2. For $b:=g(a)$ the Abelian subalgebras $\mathfrak{v}^{\prime} \subset \mathfrak{g}$ and $\rho_{b}(\mathfrak{v}) \subset \mathfrak{h o l}(M, b)$ give affinely equivalent local tube realizations. Therefore we have to show that the Abelian subalgebras $\mathfrak{v} \subset \mathfrak{g}$ and $\rho_{b}(\mathfrak{v}) \subset \mathfrak{h o l}(M, b)$ give globally affinely equivalent tube realizations of the germs $(M, a)$ and $(M, b)$. To begin with let $E$ and $V$ be the vector spaces underlying $\mathfrak{e}$ and $\mathfrak{v}$, compare with the proof of Proposition 4.3. Then the locally biholomorphic map $\psi: E \rightarrow Z, \xi \mapsto \exp (\xi)(a)$, is the universal covering of an open subset $O \subset Z$ with $Z \backslash O$ analytic in $Z$. Denote by $T$ the connected component of $\psi^{-1}(M)$ that contains the origin of $E$. By Lemma 2.2 the intersection $M \cap O$ is connected, that is, there is a point $c \in T$ with $\psi(c)=b$. Now $T$ is a tube submanifold of $E$ and the tube germ $(T, 0)$ is affinely equivalent to the tube realization of $(M, a)$ given by $\mathfrak{v} \subset \mathfrak{h o l}(M, a)$. Also the tube germ $(T, c)$ is affinely equivalent to the tube realization of $(M, b)$ given by $\rho_{b}(\mathfrak{v}) \subset \mathfrak{h o l}(M, b)$. This proves the claim. 
Corollary 6.4. In case $M$ in Proposition 6.3 is closed in $Z$, the tube realization of $(M, a)$ given by $\mathfrak{v}$ is affinely equivalent to the germ $(T, 0)$ with $T \subset E$ a suitable closed tube submanifold containing the origin. In other words, the germ $(T, 0)$ has a closed globalization $\hat{T}$ in $E$.

Proof. With the notation of the proof for Proposition 6.3 the intersection $M \cap O$ is closed in $O$. Hence also $T \subset E$ is closed.

Since every $M=S_{p, q}^{m}$, see (5.1), is closed in $Z=\mathbb{G}$ and the assumptions of Proposition 6.3 are satisfied for $M \subset Z$, we have: Every tube submanifold of $\mathbb{C}^{r}$ locally $C R$-equivalent to $S_{p, q}^{m}$ extends to a closed tube submanifold of $\mathbb{C}^{r}$ with the same property. By Section $5 S_{p, q}^{m}$ has a local tube realization only in case $m=1$, and for this special situation the statement is already contained in [14].

In case the manifold $M$ is not closed in $Z$ the globalization of a local tube realization for $M$ may be no longer closed in $E$. For a typical example compare with the lines following (9.4).

\section{The subgroup $\operatorname{Glob}(M, a) \subset \operatorname{Aut}(\mathfrak{h o l}(M, a))$}

In certain cases also the converse of Proposition 6.3 is true. Let us denote for $\mathfrak{g}=\mathfrak{h o l}(M, a)$ by

$\operatorname{Glob}(M, a) \subset$ Aut $(\mathfrak{g})$ the subgroup generated by

$\operatorname{Int}(\mathfrak{g}) \quad$ together with $\quad \operatorname{Ad}(\operatorname{Aut}(\mathrm{M}, \mathrm{a}))=\left\{\mathrm{g}_{*}: \mathrm{g} \in \operatorname{Aut}(\mathrm{M}, \mathrm{a})\right\}$.

Clearly, Int $(\mathfrak{g})$ is a connected subgroup of $\operatorname{Glob}(M, a)$ and coincides with the connected identity component of Aut $(\mathfrak{g})$ if $\mathfrak{g}$ is semi-simple. For the complex manifold $Z$ and the CR-submanifold $M \subset Z$ we will need the following

Condition P: Every CR-isomorphism of germs $(M, a) \rightarrow(M, b)$ with $a, b \in M$ extends to an automorphism $g \in \operatorname{Aut}(Z)$ with $g(M)=M$.

Condition Q: There exists an antiholomorphic automorphism $\tau$ of $Z$ with $\tau(M)=M$.

Notice that if Conditions $\mathrm{P}$ and $\mathrm{Q}$ are satisfied for $M \subset Z$ simultaneously then also every anti-CR-isomorphism of germs $\theta:(M, a) \rightarrow(M, b), a, b \in M$, extends to an antiholomorphic automorphism $\theta$ of $Z$ leaving $M$ invariant. Indeed, for $c:=$ $\tau(b)$ the CR-isomorphism $\tau \circ \theta:(M, a) \rightarrow(M, c)$ extends to a $g \in$ Aut $(Z)$ with $g(M)=M$. But then $\tau^{-1} \circ g$ is the antiholomorphic extension of $\theta$ to $Z$.

Theorem 7.1. Let $Z$ be a compact complex manifold and $M \subset Z$ a homogeneous generically embedded closed CR-submanifold satisfying condition $P$. Then, given $a \in M$, any two local tube realizations of the germ $(M, a)$ given by the Abelian Lie subalgebras $\mathfrak{v}, \mathfrak{v}^{\prime} \subset \mathfrak{g}$ are globally affinely equivalent if and only if $\mathfrak{v}=\lambda\left(\mathfrak{v}^{\prime}\right)$ for some $\lambda \in \operatorname{Glob}(M, a)$. Furthermore, the Lie algebra $\mathfrak{g}:=\mathfrak{h o l}(M, a)$ has finite dimension. 
Proof. Aut $(Z)$ is a complex Lie group in the compact-open topology with Lie algebra $\mathfrak{a u t}(Z)=\mathfrak{h o l}(Z)$ since $Z$ is compact. Every $\xi \in \mathfrak{g}$ defines a local flow in a small open neighbourhood of $a \in M$ and thus a one parameter subgroup of Aut $(Z)$ by condition P. Therefore every such $\xi$ extends to a vector field in $\mathfrak{h o l}(Z)$ tangent to $M$. Identifying $\mathfrak{g}$ and $\mathfrak{h o l}(M)$ as before via the isomorphism $\rho_{a}$ we have $\mathfrak{g}=\mathfrak{h o l}(M) \subset \mathfrak{h o l}(Z)$. In particular, $\mathfrak{g}$ has finite dimension. Let $G \subset \operatorname{Aut}(M)$ be the subgroup generated by $\exp (\mathfrak{a} \mathfrak{u t}(M))$. Then $G$ acts transitively on $M$ since by assumption $M$ is homogeneous. Therefore every $g \in \operatorname{Aut}(M)$ is of the form $g=g_{1} g_{2}$ with $g_{1} \in G$ and $g_{2}(a)=a$. This implies

$$
\operatorname{Ad}(\operatorname{Aut}(\mathrm{M})) \subset \operatorname{Int}(\mathfrak{g}) \operatorname{Ad}(\operatorname{Aut}(\mathrm{M}, \mathrm{a}))=\operatorname{Glob}(\mathrm{M}, \mathrm{a}) .
$$

'if' In case $\lambda \in \operatorname{Ad}(\operatorname{Aut}(\mathrm{M}, \mathrm{a}))$ the Abelian Lie algebras $\mathfrak{v}, \mathfrak{v}$ ' already give affine equivalent local tube realizations of $(M, a)$ by Proposition 4.1. It is therefore enough to discuss the case $\lambda \in \operatorname{Int}(\mathfrak{g})$. But this follows immediately with Proposition 6.3.

'only if' By Corollary 6.4 there are closed tube submanifolds $T, T^{\prime}$ of $E=V^{\mathbb{C}}$ containing the origin such that $(T, 0)$ and $\left(T^{\prime}, 0\right)$ are the local tube realizations of $(M, a)$ determined by $\mathfrak{v}$ and $\mathfrak{v}^{\prime}$. Also there are locally biholomorphic mappings $\psi, \psi^{\prime}: E \rightarrow Z$ with $\psi(0)=\psi^{\prime}(0)=a$ and such that $\psi(T)$ as well as $\psi^{\prime}\left(T^{\prime}\right)$ are open in $M$, compare with the proof of Proposition 6.3. Now assume that $(T, 0)$ and $\left(T^{\prime}, 0\right)$ are globally affinely equivalent. Then there exists a complex affine automorphism $h$ of $E$ with $T=h\left(T^{\prime}\right)$ (but not necessarily with $h(0)=0$ ). By condition $\mathrm{P}$ there is a unique $g \in \operatorname{Aut}(Z)$ with

$$
g \circ \psi^{\prime}=\psi \circ h
$$

and $g(M)=M$. Put $b:=g(a)$ and $c:=h(0)$. Then $\psi(c)=b$ and $\lambda:=\operatorname{Ad}(\mathrm{g}) \in$ $\operatorname{Glob}(\mathrm{M}$, a) by $(*)$. For $\mathfrak{V}:=\{v \partial / \partial z: v \in V\} \subset \mathfrak{h o l}(E)$ we have

$$
h_{*}\left(\rho_{0}(\mathfrak{V})\right)=\rho_{c}(\mathfrak{V}), \quad \psi_{*}\left(\rho_{c}(\mathfrak{V})\right)=\rho_{b}(\mathfrak{v}) \text { and } \psi_{*}^{\prime}\left(\rho_{0}(\mathfrak{V})\right)=\rho_{a}\left(\mathfrak{v}^{\prime}\right),
$$

where $\rho_{b}$ is the restriction map, introduced just before 6.2. This implies $\rho_{b}(\mathfrak{v})=$ $\rho_{b}\left(\lambda\left(\mathfrak{v}^{\prime}\right)\right)$ by $(\dagger)$ and hence $\mathfrak{v}=\lambda\left(\mathfrak{v}^{\prime}\right)$ as desired.

An example for Theorem 7.1. As an example for a pair $M \subset Z$ satisfying all the assumptions in 7.1 we may take the complex projective space $Z=\mathbb{P}_{r}$ together with the compact homogeneous hypersurface $S=S_{p, q}^{1}$ from (5.1) as $M$, where the integers $p, q, r \geq 1$ satisfy $p+q=r+1 \geq 3$. Condition $\mathrm{P}$ for example, is satisfied by $\mathrm{i}[20$, Theorem 6]. Then $L:=\operatorname{Aut}(Z)=\operatorname{PSL}(r+1, \mathbb{C})$ and $G:=\{g \in L: g(S)=S\}$ can be canonically identified with Aut $(M)$. The real Lie group $G$ has $\left(1+\delta_{p, q}\right)$ connected components, the connected identity component $G^{0}=\operatorname{PSU}(p, q)$ is a real form of $L^{0}$. For the Lie algebras we have $\mathfrak{l}:=\mathfrak{h o l}(Z)=\mathfrak{s l}(r+1, \mathbb{C})$ with real form $\mathfrak{g}:=\mathfrak{h o l}(S)=\mathfrak{s u}(p, q)$. If we fix $a \in S$ 
and identify the Lie algebras $\mathfrak{g}, \mathfrak{h o l}(S, a)$ via the restriction operator $\rho_{a}$ we have $\operatorname{Glob}(S, a)=\operatorname{Ad}(\mathrm{G}) \cong \mathrm{G}$. In particular, $\operatorname{Glob}(S, a)=\operatorname{Int}(\mathfrak{g})$ if $p \neq q$.

Now suppose that $\mathfrak{e} \subset \mathfrak{l}$ is a complex Abelian subalgebra such that the subgroup $\exp (\mathfrak{e}) \subset L$ has an open orbit $O$ in $\mathbb{P}_{r}$. By Lemma 2.1 then $\mathfrak{e}$ has dimension $r$ and is maximal Abelian in $\mathfrak{l}$. The orbit $O$ consists of all points $c \in Z=\mathbb{P}_{r}$ with $\varepsilon_{c}(\mathfrak{e})=T_{c} Z$, and the complement $A:=\mathbb{P}_{r} \backslash O$ is the union $A=H_{1} \cup H_{2} \cup \cdots \cup H_{k}$ of $k \leq r+1$ complex projective hyperplanes $H_{j}$ in $\mathbb{P}_{r}$. Clearly, the conjugacy class of $\mathfrak{e}$ in $\mathfrak{l}$ modulo the action of $L$ depends on the $L$-orbit of $A$ in the space of all analytic subsets in $\mathbb{P}_{r}$.

Suppose in addition that $\mathfrak{e}=\mathfrak{v}^{\mathbb{C}}$ for $\mathfrak{v}:=\mathfrak{e} \cap \mathfrak{g}$ and fix a point $a \in O \cap S$. Put $E:=\mathbb{C}^{r}, V:=i \mathbb{R}^{r}$ and choose a complex linear isomorphism $\Xi: E \rightarrow \mathfrak{e}$ with $\Xi(V)=\mathfrak{v}$. Then the locally biholomorphic map $\psi: E \rightarrow O, z \mapsto \exp (\Xi z) a$, realizes $E=\mathbb{C}^{r}$ as universal cover of the domain $O$. The intersection $O \cap S$ is a closed CR-submanifold of $O$ and divides $O \backslash S$ into the two connected components $O^{ \pm}:=O \cap S^{ \pm}$. In general the pre-image $\psi^{-1}(S)$ in $E$ decomposes into several connected components which only differ by a translation in $E$. Let $T$ be one of these. Then by Corollary $6.4 T$ is a closed tube submanifold of $E$ and a covering of $O \cap S$ via $\phi$.

In the next section we will discuss the special case $p=1$.

\section{The standard sphere}

In this section we consider for fixed $r \geq 2$ the Euclidean hypersphere

$$
S:=\left\{z \in \mathbb{C}^{r}:(z \mid z)=\sum z_{k} \bar{z}_{k}=1\right\}
$$

$S$ is the boundary of the Euclidean ball $B:=\left\{z \in \mathbb{C}^{r}:(z \mid z)<1\right\}$, a bounded symmetric domain of rank 1 . We always consider $\mathbb{C}^{r}$ as domain in the complex projective space $\mathbb{P}_{r}$ by identifying the points $\left(z_{1}, \ldots, z_{r}\right) \in \mathbb{C}^{r}$ and $\left[1, z_{1}, \cdots, z_{r}\right] \in \mathbb{P}_{r}$. In this sense $S$ can also be written as

$$
S=\left\{\left[z_{0}, \cdots, z_{r}\right] \in \mathbb{P}_{r}: z_{0} \bar{z}_{0}=\sum_{k>0} z_{k} \bar{z}_{k}\right\},
$$

which is the case $p=1, q=r, m=1$ in (5.1). Every $g \in \operatorname{Aut}(S)$ extends to a biholomorphic automorphism of $\mathbb{P}_{r}$ leaving the ball $B=S^{+}$as well as the outer domain $\mathbb{P}_{r} \backslash \bar{B}=S^{-}$invariant and thus gives isomorphisms of the groups

$$
G:=\operatorname{Aut}(S) \cong \operatorname{Aut}\left(S^{ \pm}\right) \cong\left\{g \in \operatorname{Aut}\left(\mathbb{P}_{r}\right): g(S)=S\right\} \cong \operatorname{PSU}(1, r),
$$

which we identify in the following. In particular, $S$ is homogeneous and $G$ is a real form of $L:=\operatorname{Aut}\left(\mathbb{P}_{r}\right)=\operatorname{PSL}(r+1, \mathbb{C})$. It is well known that $\operatorname{Aut}_{a}(S)=$ Aut $(S, a)$ holds for every $a \in S$ and that Aut $(S, a)$ acts transitively on the ball $B$. 
In the following we describe some Abelian Lie subalgebras $\mathfrak{v} \subset \mathfrak{g}:=\mathfrak{h o l}(S)$ that lead to local tube realizations of $S$. Every vector field in $\mathfrak{l}:=\mathfrak{g}^{\mathbb{C}}=\mathfrak{h o l}\left(\mathbb{P}_{r}\right)$ is polynomial of degree $\leq 2$ in the coordinate $z=\left(z_{1}, \ldots, z_{r}\right)$ of $\mathbb{C}^{r}$ and

$$
\mathfrak{g}=\left\{(\alpha+z u-(z \mid \alpha) z) \partial / \partial z: \alpha \in \mathbb{C}^{r}, u \in \mathfrak{u}(r)\right\} .
$$

With $E:=\mathbb{C}^{r}$ and $V:=i \mathbb{R}^{r}$ we start with an arbitrary but fixed $\alpha \in V$ and consider the Abelian subalgebra of $\mathfrak{g}$

$$
\mathfrak{v}:=\mathbb{R}(\alpha-(z \mid \alpha) z) \partial / \partial z \oplus\{z u \partial / \partial z: u \in \mathfrak{u}(r) \text { diagonal with } \alpha u=0\} .
$$

Then $\mathfrak{e}:=\mathfrak{v}^{\mathbb{C}} \subset \mathfrak{l}$ has an open orbit $O \subset \mathbb{P}_{r}$ and, fixing a complex linear isomorphism $\Xi: \mathbb{C}^{r} \rightarrow \mathfrak{e}$ as at the end of the preceding section, we get the universal covering map $\phi: \mathbb{C}^{r} \rightarrow O$.

In case $\alpha=0$ we have $O=\left(\mathbb{C}^{*}\right)^{r}$ and $\phi(z)=\left(e^{z_{1}}, \ldots, e^{z_{r}}\right)$ can be chosen. Then $T:=\phi^{-1}(S)=F+i \mathbb{R}^{r}$ is the tube with base

$$
F=\left\{x \in \mathbb{R}^{r}: e^{2 x_{1}}+e^{2 x_{2}}+\cdots+e^{2 x_{r}}=1\right\} .
$$

With $e^{2 x_{1}}-1=2 e^{x_{1}} \sinh x_{1}$ it is obvious that $F$ is affinely equivalent in $\mathbb{R}^{r}$ to the hypersurface

$$
\Pi_{-}:=\left\{x \in \mathbb{R}^{r}: \sinh x_{1}=\sum_{k>1} e^{x_{k}}\right\}
$$

occurring in Theorem 2 of [7]. Notice that $\mathbb{P}_{r} \backslash O=H_{0} \cup H_{1} \cup \cdots \cup H_{r}$ is the union of $r+1$ projective hyperplanes in general position with $H_{1}, \ldots, H_{r}$ intersecting $S$ transversally and $H_{0}$ not meeting $S$.

In case $\alpha=(i, 0, \ldots, 0)$ we have $O=\left\{[z] \in \mathbb{P}_{r}:\left(z_{0}^{2}+z_{1}^{2}\right) z_{2} z_{3} \cdots z_{r} \neq 0\right\}$ and $\phi$ can be chosen as

$$
\phi(z)=\left[\cos z_{1}, \sin z_{1}, e^{z_{2}}, \ldots, e^{z_{r}}\right] \text { for all } z \in \mathbb{C}^{r} .
$$

$\phi^{-1}(S)$ has a countable number of connected components which differ by a translation in $\mathbb{R}^{r}$. One of them is the tube $T:=F+i \mathbb{R}^{r}$ with base

$$
F=\left\{x \in \mathbb{R}^{r}: 2\left(\sin x_{1}\right)^{2}+\sum_{k>1} e^{2 x_{k}}=1,\left|x_{1}\right|<\pi 4\right\} .
$$

With $2\left(\sin x_{1}\right)^{2}=1-\cos 2 x_{1}$ it is clear that $F$ is linearly equivalent in $\mathbb{R}^{r}$ to

$$
\Pi_{+}:=\left\{x \in \mathbb{R}^{r}: \cos x_{1}=\sum_{k>1} e^{x_{k}},\left|x_{1}\right|<\pi / 2\right\}
$$

from [7]. Here $\mathbb{P}_{r} \backslash O$ again is the union of $r+1$ projective hyperplanes in general position, but all of them intersect $S$ and two even tangentially. Figure 8.1 depicts 


\section{Figure 8.1.}

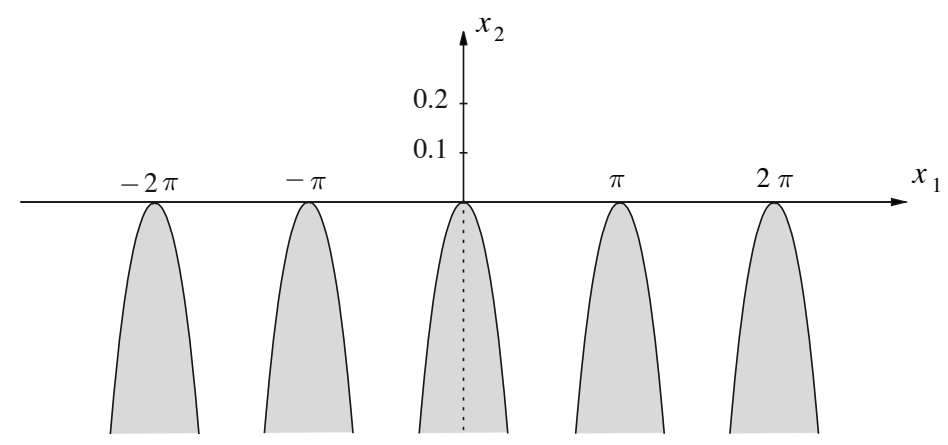

in case $r=2$ the base of $\Pi_{+}$as the boundary of the 'central' gray domain in $\mathbb{R}^{2}$. Also, the tube over the white region is the universal cover of $S^{-} \cap O$, and the tube over every gray region is the universal cover of $\left\{z \in B: z_{2} \neq 0\right\}$ via $\phi$.

Notice that the Abelian subalgebras $\mathfrak{v} \subset \mathfrak{g}$ giving the two tube realizations $\Pi_{ \pm}$ represent just the two conjugation classes of Cartan subalgebras of $\mathfrak{g} \cong \mathfrak{s u}(r, 1)(=$ maximal Abelian subalgebras consisting of ad-semisimple elements).

To get further local tube realizations another description of $S$ is convenient: Consider the classical Cayley transform $\gamma \in$ Aut $\left(\mathbb{P}_{r}\right)$ defined by

$$
\gamma([z]):=\left[z_{0}-z_{1}, z_{0}+z_{1}, \sqrt{2} z_{2}, \ldots, \sqrt{2} z_{r}\right] .
$$

Then the biholomorphic image $\gamma(S)$ in $\mathbb{P}_{r}$ is of the form

$$
S^{\prime}:=\gamma(S)=\left\{z \in \mathbb{C}^{r}: z_{1}+\bar{z}_{1}=\sum_{k=2}^{r} z_{k} \bar{z}_{k}\right\} \cup\{[0,1,0, \ldots, 0]\}
$$

With $\mathfrak{g}=\mathfrak{h o l}(S)$ and $\mathfrak{l}=\mathfrak{g}^{\mathbb{C}}$ as before let $\mathfrak{g}^{\prime}:=\mathfrak{h o l}\left(S^{\prime}\right)=\gamma_{*} \mathfrak{g}$. For fixed $1 \leq s \leq r$ let $\mathfrak{v}^{\prime}$ be the linear span of the vector fields

$$
i \partial / \partial z_{1}, i z_{r} \partial / \partial z_{r} \text { and } i\left(\partial / \partial z_{j}-z_{j} \partial / \partial z_{1}\right) \text { for } 1<r \leq s \text { and } s<j \leq r
$$

(written in the coordinate $z$ of $\mathbb{C}^{r}$ ). Then $\mathfrak{v}^{\prime}$ is an Abelian subalgebra of $\mathfrak{g}^{\prime}$ and $\mathfrak{e}^{\prime}:=\mathfrak{v}^{\prime} \oplus i \mathfrak{v}^{\prime} \subset \mathfrak{l}$ has the open orbit

$$
O^{\prime}:=\left\{z \in \mathbb{C}^{r}: z_{2} z_{3} \cdots z_{s} \neq 0\right\}
$$

in $\mathbb{P}_{r}$. As a consequence, $\mathbb{P}_{r} \backslash O^{\prime}$ is the union of $s$ mutually different projective hyperplanes. As $\phi^{\prime}: \mathbb{C}^{r} \rightarrow O^{\prime}$ we can choose

$$
\phi^{\prime}(z):=\left(\left(z_{1}-\frac{1}{2} \sum_{j>s} z_{j}^{2}, e^{z_{2}}, \ldots, e^{z_{s}}, z_{s+1}, \ldots, z_{r}\right)\right)
$$


and obtain the corresponding tube realization with base

$$
F_{s}:=\left\{x \in \mathbb{R}^{r}: x_{1}=\sum_{j=2}^{s} e^{2 x_{j}}+\sum_{j>s} x_{j}^{2}\right\} .
$$

$F_{S}$ is affinely equivalent to the hypersurface $\Pi_{s-1, r-1}$ in [7] and the tube $F_{s}+i \mathbb{R}^{r}$ is the universal covering of

$$
\left\{z \in S:\left(z_{1}-1\right) z_{2} \cdots z_{s} \neq 0\right\}
$$

via the map $\phi:=\gamma^{-1} \phi^{\prime}$.

So far we have obtained $r+2$ local tube realizations of $S$ which are mutually globally affinely inequivalent and closed in $\mathbb{C}^{r}$. Among these, in case $r>2$, there is precisely one affinely homogeneous one -the tube with base $F_{1}=\left\{x \in \mathbb{R}^{r}\right.$ : $\left.x_{1}=\sum_{j>1} x_{j}^{2}\right\}$. This is the unique algebraic tube realization and also the only case where $\phi: \mathbb{C}^{r} \rightarrow O$ is bijective and where $O \cap S$ is simply connected.

By [7] the examples above give, up to affine equivalence, all closed smooth tube submanifolds in $\mathbb{C}^{r}$ that are locally $\mathrm{CR}$-equivalent to the standard sphere $S=S_{1, r}^{1}$.

\section{Further examples}

Our methods work best for CR-manifolds that are homogeneous (or at least locally homogeneous). One way to get large classes of CR-manifolds of this type is as follows: Choose a connected complex Lie group $L$ acting holomorphically and transitively on a complex manifold $Z$, that is, $Z=L / P$ for a closed complex Lie subgroup $P$ of $L$. Choose furthermore a real form $G$ of $L$, that is, a connected real Lie subgroup such that $\mathfrak{l}=\mathfrak{g}^{\mathbb{C}}$ for the corresponding Lie algebras. Then for every $a \in Z$ the $G$-orbit $S:=G(a)$ is an (immersed) CR-submanifold that is generically embedded in $Z$ (since $\varepsilon_{a}(\mathfrak{l})=T_{a} Z$ ). Clearly, the cases $S$ open in $Z$ and $S$ totally real in $Z$ are not interesting in our situation since for these the local CR-structure is trivial and for every $a \in S$ there exists exactly one tube realization of $(S, a)$ up to affine equivalence.

A case well understood in the group level is when $Z$ is a flag manifold, that is, $L$ is semisimple and $P$ is a parabolic subgroup. Then, in particular, $Z$ is a compact rational projective variety. The simplest flag manifold is the complex projective space $\mathbb{P}_{r}$ of dimension $r \geq 1$. In this case we may take $L=\operatorname{SL}(r+1, \mathbb{C})$ which is the universal cover of the group Aut $\left(\mathbb{P}_{r}\right)$. The only real forms $G$ of $L$ having an orbit in $\mathbb{P}_{r}$ that is neither open nor totally real are, up to conjugation, the subgroups $\mathrm{SU}(p, q)$ with $p \geq q \geq 1$ and $m:=p+q=r+1$. For the sake of completeness note that the real form $G=\mathrm{SL}(m, \mathbb{R})$ has as unique non-open orbit the real projective space $\mathbb{P}_{r}(\mathbb{R}) \subset Z$. This orbit is totally real and admits up to affine equivalence a unique closed local tube realization in $E=\mathbb{C}^{r}$, namely $\mathbb{R}^{r} \subset \mathbb{C}^{n}$. The real form 
$\mathrm{SU}(m)$ and, in case $m$ is even, also the real form $\operatorname{SL}(m / 2, \mathbb{H})$ act transitively on $\mathbb{P}_{r}$.

$\mathrm{SU}(1,1)$ is conjugate to $\mathrm{SL}(2, \mathbb{R})$ in $L$, so we assume $r>1$ in the following. Then $G$ has again a unique non-open orbit in $Z$, the compact hypersurface $S=S_{p, q}^{1}$, see 5.1. With $\gamma \in$ Aut $\left(\mathbb{P}_{r}\right)$ the Cayley transform defined in (8.2)

$$
Q:=\gamma(S) \cap \mathbb{C}^{r}=\left\{z \in \mathbb{C}^{r}: z_{1}+\bar{z}_{1}=\sum_{j=2}^{r} \varepsilon_{j} z_{j} \bar{z}_{j}\right\}, \quad \varepsilon_{j}:= \begin{cases}-1 & j \leq p \\ 1 & j>p,\end{cases}
$$

is the non-singular hyperquadric with Levi form of type $(p-1, q-1)$. Now fix an integer $d$ with $1 \leq d \leq r$. The biholomorphic automorphism

$$
\left(z_{1}, \ldots, z_{r}\right) \longmapsto\left(z_{1}+\frac{1}{2} \sum_{j=2}^{d} \varepsilon_{j} z_{j}^{2}, z_{2}, \ldots, z_{r}\right)
$$

of $\mathbb{C}^{r}$ maps $Q$ to the submanifold

$$
Q^{\prime}:=\left\{z \in \mathbb{C}^{r}: z_{1}+\bar{z}_{1}=\frac{1}{2} \sum_{j=2}^{d} \varepsilon_{j}\left(z_{j}+\bar{z}_{j}\right)^{2}+\sum_{j=d+1}^{r} \varepsilon_{j} z_{j} \bar{z}_{j}\right\} .
$$

Notice that $Q^{\prime}$ has Siegel form, see Section 10,

$$
Q^{\prime}:=\left\{(v, w) \in \mathbb{C}^{d} \oplus \mathbb{C}^{r-d}:(v+\bar{v})-F(w, w) \in C\right\},
$$

where $F(w, w):=\left(\sum_{j=1}^{r-d} \varepsilon_{d+j} w_{j} \bar{w}_{j}, 0, \ldots, 0\right) \in \mathbb{R}^{d}$ and

$$
C:=\left\{x \in \mathbb{R}^{d}: x_{1}=\frac{1}{2} \sum_{j=2}^{d} \varepsilon_{j} x_{j}^{2}\right\} .
$$

In particular, $Q^{\prime}$ is a tube manifold in case $d=r$.

The next class of flag manifolds, to which our methods can easily be applied, is given by the irreducible compact hermitian symmetric spaces $Z$. Let $L$ be the universal covering of the connected identity component of Aut $(Z)$. Then $L$ is a simple complex Lie group acting transitively on $Z$ and every real form of $L$ has finitely many orbits in $Z$ that are all generically embedded CR-submanifolds. There exists a real form $G$ of $L$ with an open orbit $D$ that is biholomorphically equivalent to a bounded symmetric domain. Suppose that $D$ is of tube type and choose a $G$ orbit $S \subset Z$ that is neither open nor totally real. Then $S$ is Levi degenerate (in fact is 2-nondegenerate) and $\mathfrak{h o l}(S)=\mathfrak{h o l}(S, a)$ is isomorphic to the Lie algebra $\mathfrak{g}$ of $G$ for every $a \in S$, compare with [18]. As a special example consider for fixed $p \geq 2$ and $m:=2 p$ the Grassmannian $Z$ of all linear subspaces of dimension $p$ 
in $\mathbb{C}^{m}$. Then $Z$ has complex dimension $n:=p^{2}, L=\operatorname{SL}(m, \mathbb{C})$ and we can take $G=\mathrm{SU}(p, p)$. Now let $E:=\mathbb{C}^{p \times p}$ be the space of all complex $p \times p$-matrices and $V:=\left\{z \in E: z^{*}=z\right\}$ the $\mathbb{R}$-linear subspace of all hermitian matrices, where $z^{*}$ is the transpose conjugate of the matrix $z$. The $G$-orbits in $Z$ are in 1-1correspondence to the cones

$$
C_{j, k}^{p}:=\{x \in V: x \text { has type }(j, k)\}, \quad j, k \geq 0 \text { and } j+k \leq p,
$$

in such a way that for every $G$-orbit $S$ with corresponding $C_{j, k}^{p}$ the tube submanifold

$$
T_{j, k}^{p}:=V+i C_{j, k}^{p} \subset E
$$

is CR-equivalent to an open dense subset of $S$, see [18]. Notice that $T_{0,0}^{p}$ is the only closed tube submanifold of $E$ among the $T_{j, k}^{p}$ in (9.4) and corresponds to the unique closed $G$-orbit in $Z$ (totally real and diffeomorphic to the unitary group $\mathrm{U}(p)$ ). On the other hand, all non-open tubes $T_{j, k}^{p}$, that is $j+k<p$, are their own globalization in the sense of Section 6. Every cone $C_{j, k}^{p}$ is an orbit of the group GL (n, $\mathbb{C}$ ) acting linearly on $V$ by $x \mapsto g x g^{*}$, that is, every tube $T_{j, k}^{p}$ is affinely homogeneous. All tubes $T_{j, k}^{p}$ with $0<j+k<p$ satisfy the conditions $\mathrm{P}$ and $\mathrm{Q}$ of Section 7 .

\section{CR-manifolds of Siegel type}

In the following we generalize the notion of a tube CR-manifold. Let $V$ be a real and $W$ a complex vector space each of finite dimension. Let furthermore $F: W \times$ $W \rightarrow V^{\mathbb{C}}$ be a $V$-hermitian (vector valued) form, that is, complex linear in the first, antilinear in the second variable and $F(w, w) \in V$ for every $w \in W$. Throughout we assume that $F$ is nondegenerate, that is, $F(w, W)=0$ implies $w=0$ for every $w \in W$. For every real-analytic submanifold $C \subset V$ and $\operatorname{Im}(x+i y):=y$ for all $x, y \in V$ then

$$
\Sigma:=\left\{(z, w) \in V^{\mathbb{C}} \oplus W: \operatorname{Im} z-F(w, w) \in C\right\}
$$

is a real-analytic CR-submanifold of $E:=V^{\mathbb{C}} \oplus W$ and is called a Siegel CRsubmanifold. The CR-geometry of $\Sigma$ is closely related to the associated tube $T:=$ $\Sigma \cap V^{\mathbb{C}}=V+i C$ in $V^{\mathbb{C}}$. The submanifold $\Sigma$ is generically embedded in $E$ and Aut $(\Sigma)$ contains the nilpotent subgroup

$$
N:=\{(z, w) \mapsto(z+v+2 i F(w, c)+i F(c, c), w+c): v \in V, c \in W\}
$$

acting by affine transformations on $E$. Obviously $\Sigma=N(T)$ if we consider $T$ in the canonical way as submanifold of $\Sigma$. The Lie algebra

$$
\mathfrak{n}=\{(2 i F(w, c)+v) \partial / \partial z+c \partial / \partial w: v \in V, c \in W\} \subset \mathfrak{a u t}(\Sigma)
$$


of $N$ is nilpotent of step $\leq 2$ and can be considered as a subalgebra of $\mathfrak{h o l}(\Sigma, a)$ with $\varepsilon_{a}\left(\mathfrak{n}^{\mathbb{C}}\right)=E$ for every $a \in \Sigma$.

In a way, the nilpotent Lie subalgebras $\mathfrak{n} \subset \mathfrak{h o l}(M, a)$ play the same role for Siegel realizations of a CR-manifold germ $(M, a)$ as the Abelian subalgebras $\mathfrak{v} \subset \mathfrak{h o l}(M, a)$ do for tube realizations.

Next we are interested in conditions guaranteeing that $\mathfrak{g}:=\mathfrak{h o l}(\Sigma, a)$ has finite dimension, where $\Sigma$ is as in (10.1). We start by recalling (see e.g. [18] for details) the

Iterated Levi kernels. Let $M$ be a CR-manifold of constant degeneracy (for instance if $M$ is locally homogeneous). Then there exists an infinite descending chain of complex subbundles

$$
H M=H^{0} M \supset H^{1} M \supset \cdots \supset H^{k} M \supset \ldots
$$

where for every $a \in M$ the fiber $H_{a}^{k} M$, the $k^{\text {th }}$ Levi kernel at $a$, is defined recursively as follows: Choose a subset $\Xi \subset \Gamma(M, H M)$ with $\varepsilon_{a}(\Xi)=H_{a} M$, where $\Gamma(M, H M)$ is the space of all smooth sections in $H M$ over $M$. For every $\eta \in \Gamma\left(M, H^{k} M\right)$ the vector $\eta_{a} \in H_{a}^{k} M$ is in $H_{a}^{k+1} M$ if and only if

$$
[\xi, \eta]_{a}+i[\xi, i \eta]_{a} \in H_{a}^{k} M \text { for all } \xi \in \Xi
$$

(this condition does not depend on the choice of $\Xi$ ). In particular, $M$ is $k$-nondegenerate at every point if and only if $H^{k} M=0$, and $k$ is minimal with respect to this property.

Lemma 10.1. Suppose that $\Sigma$ from (10.1) as well as the associated tube $T=\Sigma \cap$ $V^{\mathbb{C}}$ have constant degeneracy. Then for every $a \in T \subset \Sigma$ and every $k \geq 0$ there exists a complex linear subspace $W_{a}^{k} \subset W$ with $H_{a}^{k} \Sigma=H_{a}^{k} T \oplus W_{a}^{k}$. Furthermore, $W_{a}^{0}=W$ and $F\left(W_{a}^{k+1}, W\right) \subset H_{a}^{k} T$. In particular, $H^{k} T=0$ implies $H^{k+1} \Sigma=0$.

Proof. We extend every $\xi \in \Gamma(T, T \Sigma)$ to a smooth vector field $\widetilde{\xi} \in \Gamma(\Sigma, T \Sigma)$ by requiring that for every $c \in W$ and $\gamma \in N$ defined by $\gamma(z, w)=(z+2 i F(w, c)+$ $i F(c, c), w+c)$ we have $\widetilde{\xi}_{\gamma(z, 0)}=d \gamma_{z}\left(\xi_{z}\right)$ for all $z \in T$. If we write

$$
\xi=f(z) \partial / \partial z+g(z) \partial / \partial w
$$

with suitable smooth functions $f: T \rightarrow V^{\mathbb{C}}$ and $g: T \rightarrow W$, a simple computation shows

$$
\widetilde{\xi}=(f(z-i F(w, w))+2 i F(g(z), w)) \partial / \partial z+g(z) \partial / \partial w .
$$

From the construction it is clear that $\xi \in \Gamma\left(T, H^{k} \Sigma\right)$ implies $\widetilde{\xi} \in \Gamma\left(\Sigma, H^{k} \Sigma\right)$ for all $k \in \mathbb{N}$. Every $\xi \in \Gamma(T, T \Sigma)$ has a unique decomposition $\xi=\xi^{1}+\xi^{2}$ with $\xi^{1} \in \Gamma(T, T T)$ and $\xi^{2} \in \Gamma(T, T \times W)$. Let $\Xi$ be the space of all $\tilde{\xi} \in \Gamma(\Sigma, H \Sigma)$ where $\xi \in \Gamma(T, H \Sigma)$ has constant second part $\xi^{2}$, that is, $\xi^{2}=c \partial / \partial w$ for some 
constant vector $c \in W$. Then $\varepsilon_{a}(\Xi)=H_{a} \Sigma$ is obvious. For $k=0$ the claim is obvious. Therefore assume as induction hypothesis that the claim already holds for some fixed $k \geq 0$.

Fix an arbitrary $\eta \in \Gamma\left(T, H^{k} \Sigma\right)$. Then $\eta_{a}=(\alpha, \beta)$ with $\alpha \in H_{a}^{k}(T) \subset V^{\mathbb{C}}$ and $\beta \in W$. A simple calculation shows that for every section $\xi=h(z) \partial / \partial z+$ $c \partial / \partial w \in \Gamma(T, H T)$ with $\widetilde{\xi} \in \Xi$ there exists a vector $e \in W$ such that

$$
[\widetilde{\xi}, \widetilde{\eta}]_{a}+i[\widetilde{\xi}, i \widetilde{\eta}]_{a}=\left(\left[\xi^{1}, \eta^{1}\right]_{a}+i\left[\xi^{1}, i \eta^{1}\right]_{a}-4 i F(c, \beta) \partial / \partial z\right)+e \partial / \partial w
$$

Since $h(z)$ and $c$ can be chosen independently for $\xi$ we derive from $(*)$ and the induction hypothesis that $(\alpha, \beta) \in H_{a}^{k+1} \Sigma$ implies $\alpha \in H_{a}^{k+1} T$ as well as $F(W, \beta) \subset$ $H_{a}^{k} T$. Now consider conversely an arbitrary $\alpha \in H_{a}^{k+1} T$ and fix an $\eta \in \Gamma\left(T, H^{k+1} T\right)$ with $\eta_{a}=\alpha$. Then ( $*$ ) holds with $\beta=e=0$ for every $\xi$ with $\tilde{\xi} \in \Xi$, that is, $\alpha \in H_{a}^{k+1} \Sigma$. This completes the induction step $k \rightarrow k+1$.

As an application we state

Proposition 10.2. Let $\Sigma$ be an arbitrary Siegel submanifold as in (10.1) and $T$ the associated tube manifold. Then

(i) $\Sigma$ is holomorphically nondegenerate if $T$ has the same property.

(ii) $\Sigma$ is of finite type if $T$ has the same property or, if the set $F(W, W)$ spans the vector space $V^{\mathbb{C}}$.

Proof. (i) Assume that $T$ is holomorphically nondegenerate. To show that $\Sigma$ is holomorphically nondegenerate we only have to show that $\Sigma$ is holomorphically nondegenerate at some point, see Theorem 11.5.1 in [3]. We may therefore assume without loss of generality that $T$ is of constant degeneracy and that $H^{k} T=0$. But then, as a consequence of Lemma 10.1, there exists a domain $U \subset \Sigma$ of constant degeneracy with $H^{k+1} U=0$.

(ii) In a first step assume that $T$ is of finite type in $a \in T$. Then the vector fields in $\Gamma(T, T T)$ together with all their iterated brackets span the tangent space $T_{a} T$. For all $\xi, \eta \in \Gamma(T, T T)$ we have $\widetilde{[\xi, \eta]}=[\tilde{\xi}, \tilde{\eta}]$, where the extensions $\widetilde{\xi}, \widetilde{\eta} \in$ $\Gamma(\Sigma, T \Sigma)$ are defined as in the proof of 10.1. This shows that also $\Gamma(\Sigma, H \Sigma)$ together with its iterated brackets spans the tangent space $T \Sigma_{a}$. From $N(T)=\Sigma$ we get this property at every point of $\Sigma$.

Next assume that $F(W, W)$ spans $V^{\mathbb{C}}$. For every $c \in W$ and $\xi:=c \partial / \partial w \in$ $\Gamma(T, H \Sigma)$ then

$$
\widetilde{\xi}, \tilde{i} \xi \in \Gamma(\Sigma, H \Sigma) \text { and }[\tilde{\xi}, \tilde{i} \xi]=-4 F(c, c) \partial / \partial z
$$

Since, by assumption, the vectors $F(c, c)$ span $V, \Sigma$ is of finite type at every point of $T$ and hence also at every point of $\Sigma$. 


\section{Siegel CR-manifolds and bounded symmetric domains}

Irreducible bounded symmetric domains come in six types and for all types the following considerations could be carried out in a uniform (but more involved) approach. For simplicity we restrict our attention only to the first type and there only to those domains that are not of tube type: Fix integers $q>p \geq 1$ and denote by $Z:=\mathbb{G}_{p, q}$ the Grassmannian of all $p$-dimensional linear subspaces in $\mathbb{C}^{n}$, $n:=p+q$. Then $Z$ is a compact complex manifold of complex dimension $p q$, on which the complex Lie group $L:=\operatorname{SL}(n, \mathbb{C})$ acts transitively by holomorphic transformations in a canonical way. Because of our assumption $p \neq q$ the automorphism group Aut $(Z)$ is connected and has $L$ as universal cover. The real form $G:=\mathrm{SU}(p, q)$ of $L$ has $\left(\begin{array}{c}p+2 \\ 2\end{array}\right)$ orbits in $Z$. These can be indexed as $M_{j, k}^{p, q}$, where $j, k \geq 0$ are integers with $j+k \leq p$. Indeed, choose a $G$-invariant hermitian form $\Psi$ of type $(p, q)$ on $\mathbb{C}^{n}$ and let $M_{j, k}^{p, q} \subset Z$ be the set of all linear subspaces, on which $\Psi$ has type $(j, k)$. For instance, the open orbit $M_{p, 0}^{p, q}$ is a bounded symmetric domain biholomorphically equivalent to the operator ball

$$
B:=\left\{z \in \mathbb{C}^{p \times q}:\left(\mathbb{1}-z z^{*}\right) \text { positive definite }\right\},
$$

where the matrix space $\mathbb{C}^{p \times q}$ is embedded in $Z$ as open dense subset by identifying every $c \in \mathbb{C}^{p \times q}$ with its graph $\left\{(x, x c): x \in \mathbb{C}^{p}\right\} \subset \mathbb{C}^{n}$. In this way $M_{0,0}^{p, q}$, the unique closed $G$-orbit in $Z$, corresponds to the extremal boundary of $B$

$$
\partial_{e} B:=\left\{z \in \mathbb{C}^{p \times q}: \mathbb{1}=z z^{*}\right\},
$$

and coincides also with the Shilov boundary of $B$. Notice that this compact orbit already occurs as $S_{p, q}^{p}$ in Section 5. Using a suitable Cayley transformation $\gamma \in$ Aut $(Z)$ it can be shown that every $\gamma\left(M_{j, k}^{p, q}\right)$ in the coordinate neighbourhood $\mathbb{C}^{p \times q} \subset Z$ is the CR-submanifold of Siegel type

$$
\Sigma_{j, k}^{p, q}:=\left\{(z, w) \in \mathbb{C}^{p \times p} \oplus \mathbb{C}^{p \times(q-p)}: \operatorname{Im} z-w w^{*} \in C_{j, k}^{p}\right\},
$$

where $\operatorname{Im} z=\left(z-z^{*}\right) / 2 i$ and the cone $C_{j, k}^{p}$ is as in (9.3). For $V:=\left\{z \in \mathbb{C}^{p \times p}\right.$ : $\left.z=z^{*}\right\}$ and $W:=\mathbb{C}^{p \times(q-p)}$ the map $F: W \times W \rightarrow V^{\mathbb{C}},(v, w) \mapsto v w^{*}$, satisfies $F(w, w)=0$ only for $w=0$ and its image $F(W, W)$ contains all rank-1-matrices in $\mathbb{C}^{p \times p}$. In particular, $F(W, W)$ spans $V^{\mathbb{C}}$. Therefore, by Proposition 10.2, all Siegel manifolds (11.2) and hence all $G$-orbits in $Z$ are of finite type. Now fix a $G$ orbit $M=M_{j, k}^{p, q} \subset Z$ that is not open in $Z$, that is, $j+k<p$. Denote by $T \subset \mathbb{C}^{p \times p}$ the tube over $C_{j, k}^{p}$. Then, if $j=k=0$ the tube $T$ is totally real and hence $M \cong \partial_{e} B$ is Levi nondegenerate. In all other cases, that is $0<j+k<p$, the tube $T$ is 2 nondegenerate, compare with Theorem 4.7 in [18]. This implies with Lemma 10.1 that every such $M$ is Levi degenerate but is holomorphically nondegenerate. In particular, for every non-open $G$-orbit $M$ in $Z$ and every $a \in M$ the Lie algebra 
$\mathfrak{h o l}(M, a)$ has finite dimension and contains the simple Lie algebra $\mathfrak{g}:=\mathfrak{s u}(p, q)$ On the other hand, since $G$ has a bounded symmetric domain as orbit, for every $a \in M$ there is a local coordinate $z$ around $a \in Z$ such $a$ is given by $z=0$ and that $\mathfrak{g}^{\mathbb{C}}$ contains all translation vector fields $c \partial / \partial z$ as well as the Euler field $z \partial / \partial z$. With Proposition 3.1 in [18] it follows $\mathfrak{h o l}(M)=\mathfrak{h o l}(M, a)=\mathfrak{s u}(p, q)$ for every $a \in M$ and every $G$-orbit $M$ in $Z$ which is neither open nor closed in $Z$.

Proposition 11.1. Every G-orbit $M \subset Z$ satisfies Condition $\mathrm{Q}$ of Section 7. In case $M$ is neither open nor closed in $Z$ also Condition $\mathrm{P}$ is satisfied.

Proof. The antilinear involution $z \mapsto \bar{z}$ of $\mathbb{C}^{p \times q}$ leaves the ball $B$ in (11.1) invariant and extends to an antiholomorphic involution $\tau$ of $Z=\mathbb{G}_{p, q}$. Therefore, $\tau$ leaves invariant every $G$-orbit in $Z$. Now assume that the $G$-orbit $M$ is neither open nor closed in $Z$. Then $\mathfrak{g}:=\mathfrak{h o l}(M) \cong \mathfrak{s u}(p, q)$ and for every $a \in M$ the canonical restriction mapping $\rho_{a}: \mathfrak{g} \rightarrow \mathfrak{h o l}(M, a)$ is an isomorphism of Lie algebras. For every $a \in M$ denote by $\mathfrak{g}_{a}:=\left\{\xi \in \mathfrak{g}: \xi_{a}=0\right\}$ the isotropy subalgebra at $a$. By Proposition 2.11 in [16], $\mathfrak{g}_{a}=\mathfrak{g}_{b}$ for $a, b \in Z$ only holds if $a=b$. The group Aut $(M) \cong \operatorname{PSU}(p, q)$ is connected and for $H:=\operatorname{Aut}(M) \cup \operatorname{Aut}(M) \tau$ the homomorphism Ad : $\mathrm{H} \rightarrow$ Aut $(\mathfrak{g})$ is an isomorphism, compare with Proposition 4.5 in [16]. In particular, Aut ( $\mathfrak{g})$ has two connected components. Now suppose that $\phi:(M, a) \rightarrow(M, b)$ is either a CR-isomorphism or an anti-CR-isomorphism of germs, where $a, b \in M$ are arbitrary points. Then $\rho_{b}^{-1} \phi_{*} \rho_{a}$ is in Aut $(\mathfrak{g})$. In case $\rho_{b}^{-1} \phi_{*} \rho_{a}$ is contained in the connected identity component Int $(\mathfrak{g})$ of Aut $(\mathfrak{g})$ there exists a transformation $g \in G$ such that $\rho_{c}^{-1} \psi_{*} \rho_{a}=$ id for $c:=g(b)$ and $\psi:=g \phi:(M, a) \rightarrow(M, c)$. This implies $a=c$ and even $\psi=$ id since $\rho_{c}^{-1} \psi_{*} \rho_{a}$ leaves invariant all isotropy subalgebras $\mathfrak{g}_{x}$ for all $x \in M$ near $a$. As a consequence, $\phi$ extends to the global transformation $g^{-1} \in G$ in case $\rho_{b}^{-1} \phi_{*} \rho_{a} \in \operatorname{Int}(\mathfrak{g})$. But the case $\rho_{b}^{-1} \phi_{*} \rho_{a} \notin \operatorname{Int}(\mathfrak{g})$ cannot occur since otherwise $\rho_{e}^{-1}(\tau \phi)_{*} \rho_{a} \in$ Int for $e:=$ $\tau(b)$ by the above reasoning would imply that $\tau \phi$ is a CR-mapping, or equivalently, that $\phi$ is anti-CR.

By the above considerations we know that for every non-open $G$-orbit $M=$ $M_{j, k}^{p, q}$ in $Z$ there is an integer $1 \leq k \leq 3$ such that $M$ is $k$-nondegenerate. In case $j+k=0$ we have $k=1$, and we claim that $k=2$ in all other cases (compare also with [8]): Indeed, instead of $M$ we consider the Siegel manifold $\Sigma=\Sigma_{j, k}^{p, q}$ with $V^{\mathbb{C}}=\mathbb{C}^{p \times p}, W=\mathbb{C}^{p \times(q-p)}$ and $F(w, w)=w w^{*}$, see (11.2). With $\rho:=j+k$ we write all $z \in V^{\mathbb{C}}$ and $w \in W$ as block matrices

$$
z=\left(\begin{array}{ll}
z_{11} & z_{12} \\
z_{21} & z_{22}
\end{array}\right) \text { and } w=\left(\begin{array}{l}
w_{1} \\
w_{2}
\end{array}\right)
$$

where $z_{11} \in \mathbb{C}^{\rho \times \rho}, w_{1} \in \mathbb{C}^{\rho \times(q-p)}$ and so forth. Fix an element $a \in T=\Sigma \cap V^{\mathbb{C}}$ with $a_{r s}=0$ for $(r, s) \neq(1,1)$. Then it is known that

$$
H_{a}^{k} T=\left\{z \in V^{\mathbb{C}}: z_{r s}=0 \text { if } k+r+s>3\right\},
$$


see [18, page 480]. This implies $w_{2}=0$ for every $w \in W_{a}^{1}$ and thus $W_{a}^{2}=0$, that is, $H_{a}^{1} \Sigma \neq 0$ and $H_{a}^{2} \Sigma=0$.

The antiholomorphic involution $\theta$ of $Z$ given on $E=\mathbb{C}^{p \times q} \subset \mathbb{G}_{p, q}$ by $\theta(z)=$ $-\bar{z}$ leaves every Siegel manifold $\Sigma=\Sigma_{j, k}^{p, q}$ in (11.2) invariant and has fixed points there. For every such fixed point $a \in \Sigma$ then $T_{a}^{-\theta} \Sigma=\mathbb{R}^{p \times q}$, that is, $\vartheta_{a}(\theta)=0$ holds in this situation. Assuming in the following that $\Sigma$ is not open in $E$ we can have a local tube realization of $(\Sigma, a)$ associated with the involution $\theta$ only if there is a maximal Abelian subalgebra of $\mathfrak{g}=\mathfrak{s u}(p, q)$ with dimension $p q$. It can be shown that this is not possible if $p>1$.

\section{References}

[1] M. S. Baouendi, L. P. Rothschild and F. TReVes, CR structures with group action and extendibility of $C R$ functions, Invent. Math. 82 (1985), 359-396.

[2] M. S. BaOUEnd, H. JaCOBOWITZ and F. TREVES, On the analyticity of $C R$ mappings, Ann. of Math. 122 (1985), 365-400.

[3] M. S. Baouendi, P. Ebenfelt and L. P. Rothschild, "Real Submanifolds in Complex Spaces and Their Mappings", Princeton Math. Series, Vol. 47, Princeton Univ. Press, 1998.

[4] M. S. BAOUENDI, L. RothSCHILD and D. ZAITSEV, Equivalences of real submanifolds in complex space, J. Differential Geom. 59 (2001), 301-351.

[5] A. Boggess, "CR manifolds and the tangential Cauchy Riemann complex", Studies in Advanced Mathematics, Boca Raton, FL: CRC Press, 1991.

[6] S. S. CHERN and J. K. Moser, Real hypersurfaces in complex manifolds, Acta. Math. 133 (1974), 219-271.

[7] J. DADOK and P. YANG, Automorphisms of tube domains and spherical hypersurfaces, Amer. J. Math. 107 (1985), 999-1013.

[8] G. FeLS, Locally homogeneous finitely nondegenerate CR-manifolds, Math. Res. Lett. 14 (2007), 693-922.

[9] G. FELS and W. KAUP, CR-manifolds of dimension 5: A Lie algebra approach, J. Reine Angew. Math. 604 (2007), 47-71.

[10] G. FELS and W. KAUP, Classification of Levi degenerate homogeneous CR-manifolds of dimension 5, Acta Math. 201 (2008), 1-82.

[11] G. FELS and W. KAUP, Classification of commutative algebras and tube realizations of hyperquadrics, arXiv:0906.5549.

[12] A. V. ISAEV and M. A. MISHCHENKO, Classification of spherical tube hypersurfaces that have one minus in the Levi signature form, Math. USSR-Izv. 33 (1989), 441-472.

[13] A. V. ISAEv, Classification of spherical tube hypersurfaces that have two minuses in the Levi signature form, Math. Notes 46 (1989), 517-523.

[14] A. V. IsAEV, Global properties of spherical tube hypersurfaces, Indiana Univ. Math. J. 42 (1993), 179-213.

[15] A. V. ISAEV and W. KAUP, Regularization of local CR-automorphisms of real-analytic CR-manifolds, J. Geom. Anal. (2011), to appear.

[16] W. KAUP, On the holomorphic structure of G-orbits in compact hermitian symmetric spaces, Math. Z. 249 (2005), 797-816.

[17] W. KAUP, CR-quadrics with a symmetry property, Manuscripta Math. 133 (2010), 505-517.

[18] W. KAUP and D. ZAITSEV, On local CR-transformations of Levi degenerate group orbits in compact Hermitian symmetric spaces, J. Eur. Math. Soc. 8 (2006), 465-490.

[19] R. S. PALAIS, "A Global Formulation of the Lie Theory of Transformation Groups", Mem. Amer. Math. Soc. 1957. 
[20] N. TANAKA, On the pseudo-conformal geometry of hypersurfaces of the space of $n$ complex variables, J. Math. Soc. Japan 14 (1962), 397-429.

[21] D. ZAITSEV, On different notions of homogeneity for CR-manifolds, Asian J. Math. 11 (2007), 331-340.

Mathematisches Institut Universität Tübingen Auf der Morgenstelle 10 72076 Tübingen, Germany gfels@uni-tuebingen.de kaup@uni-tuebingen.de 